\title{
Non-Gaussian, transiently anomalous and ergodic self-diffusion of flexible dumbbells in crowded two-dimensional environments: coupled translational and rotational motions
}

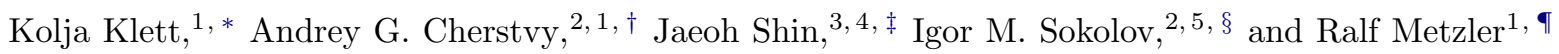 \\ ${ }^{1}$ Institute for Physics \& Astronomy, University of Potsdam, 14476 Potsdam-Golm, Germany \\ ${ }^{2}$ Institut für Physik, Humboldt-Universität zu Berlin, Newtonstraße 15, 12489 Berlin, Germany \\ ${ }^{3}$ Department of Chemistry, Rice University, Houston, Texas 7ro05, USA \\ ${ }^{4}$ Center for Theoretical Biological Physics, Rice University, Houston, Texas 7ro05, USA \\ ${ }^{5}$ IRIS Adlershof, Zum Großen Windkanal 6, 12489 Berlin, Germany
}

(Dated: September 6, 2021)

\begin{abstract}
We employ Langevin-dynamics simulations to unveil non-Brownian and non-Gaussian center-ofmass self-diffusion of massive flexible dumbbell-shaped particles in crowded two-dimensional solutions. We also study the intra-dumbbell dynamics due to the relative motion of the two constituent elastically-coupled disks. Our main focus is on effects of the crowding fraction $\phi$ and the particle structure on the diffusion characteristics. We evaluate the time-averaged mean-squared displacement (TAMSD), the displacement probability-density function (PDF) and the displacement autocorrelation function $(\mathrm{ACF})$ of the dimers. For the TAMSD at highly crowded conditions of dumbbells, e.g., we observe a transition from the short-time ballistic behavior, via an intermediate subdiffusive regime, to long-time Brownian-like spreading dynamics. The crowded system of dimers exhibits two distinct diffusion regimes distinguished by the scaling exponent of the TAMSD, the dependence of the diffusivity on $\phi$, and the features of the displacement-ACF. We attribute these regimes to a crowding-induced transition from a viscous to a viscoelastic diffusion medium upon growing $\phi$. We also analyze the relative motion in the dimers, finding that larger $\phi$ suppress their vibrations and yield strongly non-Gaussian PDFs of rotational displacements. For the diffusion coefficients $D(\phi)$ of translational and rotational motion of the dumbbells an exponential decay with $\phi$ for weak and a power-law $D(\phi) \propto\left(\phi-\phi^{\star}\right)^{2.4}$ for strong crowding is found. A comparison of simulation results with theoretical predictions for $D(\phi)$ is discussed and some relevant experimental systems are overviewed.
\end{abstract}

\section{INTRODUCTION}

\section{A. Historical prologue}

In 1827, R. Brown observed under a microscope the erratic motion of micron-sized granules released from pollen grains ${ }^{\mathbb{T}}$. The paradigmatic stochastic processnamed later Brownian motion (BM) - was physically interpreted by A. Einstein ${ }^{\square}$ in his annus-mirabilis 1905 paper (and in later studies $\left.{ }^{[3,4}\right)$. The probability-density function (PDF) $P(x, t)$ of one-dimensional BM with the long-time diffusion coefficient $D$ satisfies the diffusion equation, $\frac{\partial P(x, t)}{\partial t}=D \frac{\partial^{2} P(x, t)}{\partial x^{2}}$, introduced in 1855 by A. Fick ${ }^{5}$. The derivation of $\mathrm{BM}^{[2}$ is based on the assumptions of independence of motion of a given particle from other particles and of identically distributed particle displacements that become independent after a finite correlation time (yielding a finite second moment). The era of "random walks" started with the papers of L. Bachelier $^{[6}$, W. Sutherland ${ }^{[7}$, A. Einstein ${ }^{[}$, K. Pearson ${ }^{8}$, and M. Smoluchowski ${ }^{\mathbf{9}}$.

The solution of the diffusion equation for a single particle at position $x$ with P. Dirac's $\mathrm{s}^{\mathbb{1 0}}$ delta-function initial condition $P\left(x, t_{0}=0\right)=\delta\left(x-x_{0}\right)$ is the Gaussian PDF [after C. Gauß $\left.{ }^{[1]}\right]$ at time $t$,

$$
P(x, t)=\exp \left[-\frac{\left(x-x_{0}\right)^{2}}{4 D t}\right] / \sqrt{4 \pi D t} .
$$

This PDF yields the linear growth of the mean-squared displacement (MSD),

$$
\left\langle x^{2}(t)\right\rangle=\int x^{2} P(x, t) d x=2 D t,
$$

a hallmark of BM. All ensemble-averaged quantities are denoted by angular brackets below. The diffusivity of a spherical tracer of radius $R$ at the absolute temperature $\mathcal{T}$, denoted $D(\mathcal{T})$, is linked to the (dynamical) fluid viscosity $\eta$ via the Einstein-Smoluchowski-Stokes relation $^{[2,9}$,

$$
D=k_{B} \mathcal{T} / \gamma
$$

where $\gamma=6 \pi \eta R$ is the drag constant and $k_{B}$ is the Boltzmann constant.

In 1908, P. Langevin derived Eqs. (2) and (3) using a microscopic inherently stochastic approach ${ }^{12}$ for a spherical particle of finite mass $m$. Starting from I. Newton's second law ${ }^{[3]}$ - from his annus mirabilis 1666 (marked also by the "Great Plague") — using G. Stokes' dragintroduced in $1851^{144}$ for slowly moving bodies in viscous fluids - and the random force $\xi(t)$ [taken to be centered white Gaussian noise], the stochastic differential equation was postulated,

$$
m \frac{\mathrm{d}^{2} x(t)}{\mathrm{d} t^{2}}=-\gamma \frac{\mathrm{d} x(t)}{\mathrm{d} t}+\xi(t),
$$

(named later after Langevin). As Eq. (团) takes inertia into account, at short times when $t \ll \tau_{0}$, where

$$
\tau_{0}=m / \gamma
$$




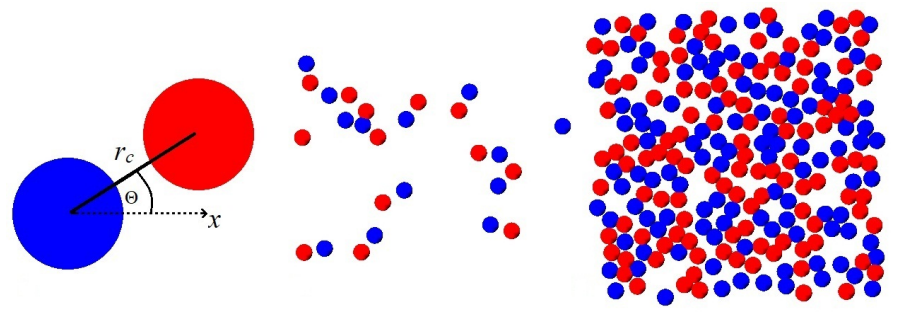

(A)

(C)
FIG. 1: (A) Dumbbell-shaped dimer of our simulation model. (B) Diluted system of dimers at crowding fraction $\phi=0.05$. (C) Highly crowded system of dumbbells at $\phi=0.45$. We refer the reader to the supplementary video files (with 360 simulation steps corresponding to the duration $0.18 \times \delta \tau$ per one second of the videos) illustrating the dynamics of dumbbells at $\phi=\{0.15,0.35,0.65\}$.

is the characteristic time of momentum relaxation for translational motion [the damping constant is $1 / \tau_{0}$ ], a ballistic behavior of the MSD,

$$
\left\langle x^{2}(t)\right\rangle \approx\left(k_{B} \mathcal{T} / m\right) t^{2},
$$

emerges, while the linear MSD growth (2) sets in at long times, for $t \gg \tau_{0}{ }^{115-177}$. The features of BM executed by ellipsoidal particles was first theoretically considered by F. Perrin in Refs, $\stackrel{18, \pi 9}{\text {, }}$

\section{B. Anomalous diffusion, macromolecular crowding, and spreading of non-spherical particles}

Standard BM has been generalized in numerous mathematical models of anomalous diffusion over the last decades ${ }^{20-24}$. These models feature a characteristic nonlinear growth of the MSD with time $\sqrt{24-\sqrt{29}}$,

$$
\left\langle x^{2}(t)\right\rangle=2 D_{\beta} t^{\beta} .
$$

Here, the generalized diffusion coefficient is $D_{\beta}$ and the anomalous-diffusion scaling exponent is $\beta$. The dispersion law for BM follows from (7) for $\beta=1$, while subdiffusion corresponds to $0<\beta<1$, superdiffusion to $\beta>1$ (for actively driven systems), and hyperdiffusionoften found in highly nonequilibrium and dynamically "accelerating" systems - to $\beta>2$. Following growing evidence for experimental systems, a number of new statistical models were proposed in recent years to describe anomalous diffusion ${ }^{[24-[27}$. The list includes fractional $\mathrm{BM}^{[30-[34}$, generalized/fractional Langevin equation motion $^{[35-[37}$, scaled $\mathrm{BM}^{[38-47]}$, extensions of continuoustime random-walk models ${ }^{[3,42-41,-48}$, and heterogeneous diffusion processes $33,499,50$ *

\footnotetext{
*As an example, superdiffusion detected in the cytoplasm of Acanthamoebae was attributed to persistent active motions caused by
}

In the cell cytoplasm, the average degree of crowding is the volume fraction taken from the aqueous phase ${ }^{[27,53-77]}$ by proteins, diverse macromolecules, complexes, and cell organelles. In cells, the fraction of crowders $\phi$ can reach $\phi \sim 30 \ldots 40 \%$ with concentrations of various proteins and macromolecules up to $\sim 300 \ldots 400 \mathrm{mg} / \mathrm{ml}$. In colloidal systems, the crowding fractions can be even higher triggering liquid-glass phase transitions ${ }^{[2-76]}$ (see Refs. ${ }^{[7,78}$ for glass transitions in $\left.2 \mathrm{D}\right)$.

Space-filling and crowding in excluded-volume solutions - causing effective "labyrinthization" and "porosityzation" of the diffusive environment (also with tracer-crowders interactions $\left.{ }^{[79}\right)$ - is considered as a cause of subdiffusion of passive particles in densely crowded systems. This is interlinked to implications of viscoelasticity in space-restricted surroundings and in the presence of multiple boundaries, nontrivially affecting the tracer diffusion, even in the limit of transport with small diffusion coefficients and particle speeds.

Normal and anomalous diffusion in the crowded solutions of anisotropic and/or non-spherical - as well as of spherical but non-isotropically interacting ${ }^{[01,81}$ molecules was examined in a number of studies ${ }^{82-103}$, including some recent experimental investigations ${ }^{96,09,104}$. The diffusive properties of molecules of dumbbell-, ellipsoid-, and rod-like shape ${ }^{[82,, 96,07,100,101,104-109}$, protein molecules ${ }^{[10,171]}$, active and passive dimers with anisotropic mobility $\frac{89,102,106,112-1144}{10}$, self-propelled massive particles with time-dependent mass ${ }^{109}$, colloidal dimers and flexible multimers ${ }^{[15,176}$, stiff self-propelled

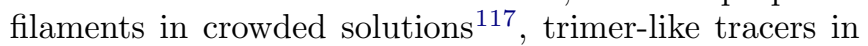
$2 \mathrm{D}^{93,95}$ and tetrahedral patchy particles in $3 \mathrm{D}^{\sqrt{81,}, 118}$, as well as the dynamics of elongated particles with fluctuating and oscillating shapes ${ }^{\square 19-1211}$ were studied recently as well.

A dumbbell-shaped dimer, see Fig. \A, is an example of a simple linear crowder for which the effects of interparticle interactions and shape variations on the translational and rotational diffusion dynamics can be examined. Under conditions of dense packing, the motion of a given crowder is no longer independent of that of the neighboring crowders, see Fig. TC. This invalidates one of the assumptions for BM. As a result, subdiffusion can emerge (as we show below) due, in part, to anticorrelations of consecutive displacements of the particles in such a highly crowded system.

The examples of physical systems where diffusion of anisotropic crowders is of importance include anisotropic crowders in the cell cytoplasm 55,63 , rod-shaped grains ${ }^{122}$ in various granular gases ${ }^{[23-125,}$, molecular components of ultra-dense ionic liquids ${ }^{126,127}$ and other complex fluids ${ }^{98,128}$, diffusion of proteins and enzymes on/in

myosin-II motors and cell locomotion 5 [5]. Conversely, subdiffusion is often associated with the passive motion of tracers in crowded, polymer containing, "restrictive" environments giving rise to antipersistent motion, such as the cytoplasm of biological cells 52 . 


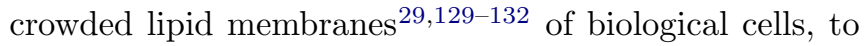
mention a few.

This paper is organized as follows. We start in Sec. 미 by describing the model potentials and simulation scheme of dense 2D solutions of dumbbell-shaped crowders. In Sec. WI the main results of our computer simulations are presented and examined based on a number of standard statistical quantifiers. The latter include the MSD, the time-averaged MSD (TAMSD), the exponents and transport coefficients, the PDFs, the non-Gaussianity parameter, and various autocorrelation functions (ACFs). In Sec. $\mathbb{\text { IV }}$ some physical applications are discussed. In Apps. $\mathbb{A}$ and $\mathbb{B}$, correspondingly, some details of the simulation scheme and some auxiliary figures are presented.

\section{PHYSICAL MODEL}

\section{A. Interaction potentials and main equations}

We consider an ensemble of identical 2D dumbbellshaped dimers in 2D solution. Each dimer, see Fig. ПIA, consists of two disks of diameter $\sigma$ connected by an elastic spring represented by the harmonic potential,

$$
U_{\text {harm }}(r)=k\left(r-r_{0}\right)^{2} / 2 .
$$

Here, $r_{0}=1.5 \sigma$ is the equilibrium disk-to-disk distance and $k=100 \times k_{B} \mathcal{T} / \sigma^{2}$ is Hooke's constant. In addition, the standard Lennard-Jones 6-12 potential is used to parameterize the interactions between the disks, ${ }^{\dagger}$

$$
U_{\mathrm{LJ}}(r)=-4 \epsilon\left[(\sigma / r)^{6}-(\sigma / r)^{12}\right] .
$$

The parameter $\epsilon$ sets the interaction strength $\left(\epsilon=1 k_{B} \mathcal{T}\right.$ below). The minimum of the interaction energy $U_{\min }$ is at $r_{\max }=2^{1 / 6} \sigma \approx 1.12 \sigma$. The repulsive branch of the Lennard-Jones potential (9) at $r<r_{\max }$ is used in the simulations, yielding the Weeks-Chandler-Anderson potential $^{137-139}$. It is given by expression (19) shifted up by $U_{\min }$ at $r<r_{\max }$ and by $U=0$ at $r>r_{\max }$. As $r_{0}>r_{\max }$ the disks of the neighboring dimers can get closer than those of the same one.

The respective Langevin equation for the position of the $i$ th monomer-disk $\mathbf{r}_{i}(t)$ is given by

$$
\begin{aligned}
m \frac{\mathrm{d}^{2} \mathbf{r}_{i}(t)}{\mathrm{d} t^{2}} & =-\gamma \frac{\mathrm{d} \mathbf{r}_{i}(t)}{\mathrm{d} t}-\sum_{\substack{j=1, j \neq i}}^{2 N} \nabla\left(U_{\mathrm{LJ}}\left(r_{i j}\right) \theta\left(2^{1 / 6} \sigma-r_{i j}\right)\right) \\
& -\nabla\left(U_{\mathrm{harm}}\left(r_{i^{\prime} i^{\prime \prime}}\right)\right)+\boldsymbol{\xi}_{i}(t) .
\end{aligned}
$$

\footnotetext{
${ }^{\dagger}$ Here, the $r^{-12}$-term models a strong short-range repulsion [stemming from the quantum-mechanical exclusion in overlapping electron clouds], whereas the $r^{-6}$-term mimics a weak long-range vander-Waals-like attraction, as in the models of diatomic molecules and chemical bonds $\sqrt{13.3-\pi .36}$.
}

Here, $\theta(x)$ is the unit-step function, the sum runs over all $2 N$ disks of $N$ dumbbells, and $\boldsymbol{\xi}(t)$ is Gaussian white noise with zero mean $\langle\boldsymbol{\xi}(t)\rangle=0$ and delta-function ${ }^{[140}$ correlations

$$
\left\langle\boldsymbol{\xi}(t) \boldsymbol{\xi}\left(t^{\prime}\right)\right\rangle=4 \gamma k_{B} \mathcal{T} \delta_{x y} \delta\left(t-t^{\prime}\right) .
$$

Here, $\delta_{x y}$ is Kronecker's delta-symbol that renders the noise in ([1]) independent for $x$ and $y$ components. The noise strength is coupled to the temperature and the friction coefficient via the fluctuation-dissipation relation. The distance $r_{i^{\prime} i^{\prime \prime}}$ in Eq. (피) is the separation of the two disks $i^{\prime}$ and $i^{\prime \prime}$ in the same $i$ th dimer.

\section{B. Self-diffusivity and crowding}

All dimers are located in a square box of area $L^{2}$, but due to periodic boundary conditions their motion is effectively unconfined. For $N$ dimers occupying the area $A=$ $2 \pi(\sigma / 2)^{2}$ the packing fraction $\phi$ in the box is $\phi=N A / L^{2}$. $\ddagger$ We fix the system size to $L=20 \sigma$ for simulations to be manageable on a PC without causing artificial selfinteractions. The number $N$ of dimers and the respective $\phi$ fractions used in our simulations as listed in Tab. [I. As our main interest is to study the consequences of crowding and thus fluid inertia ${ }^{\sqrt{4.5-}-\mathbb{1 4 7}}$ and hydrodynamic

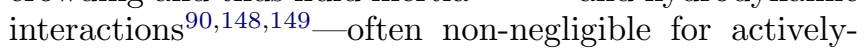
driven and quickly moving particles - are not explicitly included in our model of the passively diffusing dimers.

$$
\begin{array}{c|c|c|c|c|c|c|c|c}
N= & 1 & 13 & 38 & 63 & 89 & 115 & 140 & 166 \\
\hline \phi \approx & 0.004 & 0.05 & 0.15 & 0.25 & 0.35 & 0.45 & 0.55 & 0.65
\end{array}
$$

TABLE I: Numbers of dimers $N$ and the corresponding packing fractions $\phi$ used in simulations.

\section{Simulation algorithms and system equilibration}

We set $\gamma=1$ in most of the computer simulationsat $m=1$ and $k=100$ a free isolated dimer has a small damping ratio and, thus, executes underdamped motion - and compute time series of length $T_{\text {sim }}=10^{8} \times$ $\Delta t$. We use the standard combination of parameters ${ }^{150}$ for defining the characteristic time scale ${ }^{93}$,

$$
\delta \tau=200 \times \Delta t=\sigma \sqrt{m /\left(k_{B} \mathcal{T}\right)} \approx 1 \mathrm{~ns},
$$

where the disk diameter is set to $\sigma=6 \mathrm{~nm}$, the mass $m$ is estimated from the average molecular weight of

$\ddagger$ Note that the maximal packing fraction of identical disks in $2 \mathrm{D}$ is $\frac{141-143}{143} \phi_{\max }=\pi /(2 \sqrt{3}) \approx 0.9069$ (the hexagonal packing), while in $3 \mathrm{D}$ the maximal packing density of identical spheres is (see, e.g., Ref. ${ }^{[44}$ ) $\phi_{\max }=\pi /(3 \sqrt{2}) \approx 0.7405$ (the so-called "cannon-ball" packing). 
crowding molecules in the nucleus ${ }^{[5]}$ (with $\mathrm{MW} \approx 68$ $\left.\mathrm{kDa}^{601}\right)$, and the temperature is set to $\mathcal{T}=272.15 \mathrm{~K}$. The length of simulated traces is $T_{\text {sim }}=0.5 \mathrm{~ms}$. The latter is, thus, comparable to typical time-scales in computersimulations data-sets and single-particle-tracking experiments. All distances and times are expressed below in units $\sigma$ and $\delta \tau$, respectively.

We equilibrate the system for the time $T_{\text {eq }}$ before the actual measurements start, see Fig. 57] for a detailed scheme. For a dimer of size $R_{\mathrm{cr}}$ and diffusivity $D(\phi)$ we define $T_{\text {cr }}$ as the typical time for a dimer to diffuse over a distance of its own size ${ }^{9.3}$, i.e. $T_{\mathrm{cr}}=R_{\mathrm{cr}}^{2} / D(\phi)$. To allow proper mixing of the dimers in the course of simulations, we equilibrate for $T_{\text {eq }}=2 \times 10^{4} \times \delta \tau$ for all $\phi$ values (so that $T_{\text {eq }} \geq 10^{2} \times T_{\text {cr }}$ ).

At the start of the simulations, the dimers are placed in a regular pattern and the disks of each dimer are further than $r_{0}$ apart. The memory of these initial conditions is lost in the results presented below after equilibration of the system, for all $\phi$ values. $\S$ After equilibrating the system for $T_{\text {eq }}$, at every time-step we record four physical observables for each dimer: the $x$ and $y$ coordinates of its center of mass $(\mathrm{COM})$, denoted as $x_{\mathrm{COM}}(t)$ and $y_{\mathrm{COM}}(t)$, the angle $\Theta(t)$ between its axis and the $x$-axis, see Fig. पA, and the relative distance between the disks in the dimer, $d(t)$. Their displacements separated by a lag time $\Delta$ are denoted as

$$
\delta_{\{x, y\}}(t, \Delta)=\{x, y\}_{\mathrm{COM}}(t+\Delta)-\{x, y\}_{\mathrm{COM}}(t),
$$

$$
\delta_{r}(t, \Delta)=\Theta(t+\Delta)-\Theta(t), \delta_{d}(t, \Delta)=d(t+\Delta)-d(t) .
$$

By symmetry, the behaviors of $\delta_{x}$ and $\delta_{y}$ are statistically identical, so we present only the results for the $x$ coordinate below.

We employ the velocity Verlet algorithm ${ }^{150}$ to simulate

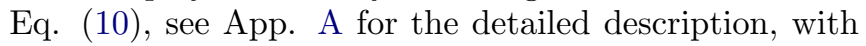
the integration time step $\Delta t=0.05 \times \delta \tau$ and the number of iterations $10^{8}$. We start measuring after the system reaches the equilibrium state. In Fig. [5] we show a simplified flow-chart of simulations: after the initial setup, the integration loop is executed and Eqs. (A.5) are repeatedly evaluated.

\section{MAIN RESULTS}

Below the data from our computer simulations are analyzed and the diffusive properties of the dumbbell-shaped dimers are examined for both translational and rotational motion. We employ the standard physical observables

\footnotetext{
$\S$ Note that, as in Ref. ${ }^{93}$, the time $T_{\text {cr }}$ grows with $\phi$ because $D(\phi)$ is a decreasing function of the crowding fraction $\phi$ (as we demonstrate below). Therefore, the most crowded system is taken below as a "reference" for the characteristic diffusion time, namely $T_{\mathrm{cr}}=$ $T_{\mathrm{cr}}\left(\phi_{\max }=0.65\right)$.
}
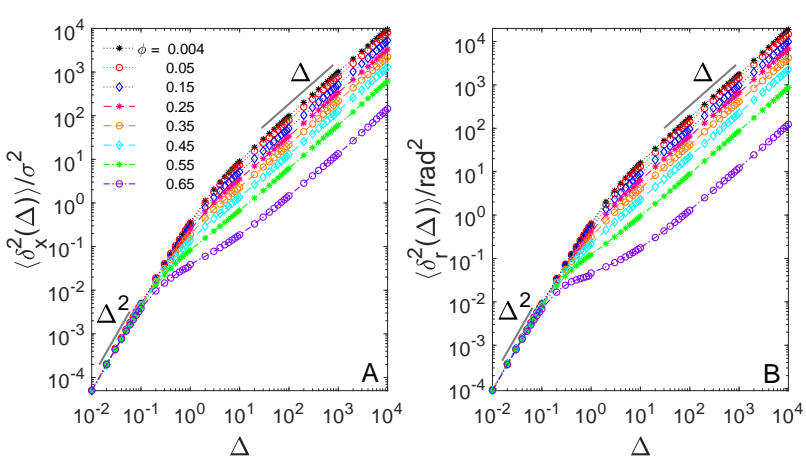

FIG. 2: (A) Translational TAMSD in units of $\sigma^{2}$ and (B) rotational TAMSD in units of $\operatorname{rad}^{2}$ of the dumbbell-shaped crowders given by Eq. (14) plotted versus lag time for varying crowding fractions $\phi$. The ballistic and linear asymptotes at short and long times, respectively, are shown in each panel as the thin lines. Parameters: the mass of the disk is $m=1$, the friction coefficient is $\gamma=1$ yielding the velocity-relaxation time $\tau_{0}=1 \times \delta \tau$, the strength of the potential is $\epsilon=1 k_{B} \mathcal{T}$, the trace length is $T=5 \times 10^{5}$, and the averages are computed for an ensemble of $N=40$ trajectories. The lag time $\Delta$ and the trajectory length $T$ are given in units $\delta \tau \approx 1 \mathrm{~ns}$ in this and all other plots. The values for the crowding fractions $\phi$ are provided in the legend.

such as the MSD and the TAMSD, their respective scaling exponents and generalized diffusivities, the displacement PDFs, and the ACFs.

\section{A. TAMSD and MSD}

We compute the translational TAMSD for the COM positions (denoted as $\overline{\delta_{x}^{2}}$ ) and the rotational TAMSD for the angles $\Theta$ (denoted as $\overline{\delta_{r}^{2}}$ ) of the $i$ th dimer as the sliding averages along respective time series recorded from simulations of Eq. ([0]) via the standard definition ${ }^{[24,25,253}$

$$
\overline{\delta_{i,\{x, r\}}^{2}(\Delta)}=\frac{1}{T-\Delta} \int_{0}^{T-\Delta} \delta_{i,\{x, r\}}^{2}(t, \Delta) \mathrm{d} t .
$$

Here, $T$ is the total length of the recorded trajectories

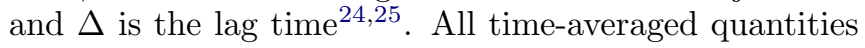
are denoted by an overline below. The mean TAMSD after averaging over $N$ trajectories is

$$
\left\langle\overline{\delta^{2}(\Delta)}\right\rangle=\frac{1}{N} \sum_{i=1}^{N} \overline{\delta_{i}^{2}(\Delta)}
$$

In Fig. 2 we show the behavior of the computed translational and rotational TAMSDs of a single dimer versus the lag time $\Delta$ for varying crowding fraction $\phi$. Initially, for lag times $\Delta \lesssim 0.1 \times \delta \tau$, the diffusion of dimers is found to be ballistic and the magnitude of the mean TAMSDs is almost the same for all $\phi$ fractions examined: in this short-time regime the dimers perform 
free motion without collisions, with the average speed $\sqrt{\left\langle v^{2}\right\rangle_{\text {eq }}}=\sqrt{k_{B} \mathcal{T} / m}$ given by Eq. (目). With increasing $\phi$ this regime shrinks to progressively shorter times. The ballistic regime at short times is also detected in simulations of diffusion of a single dimer (corresponding to $\left.\phi_{\min }=0.004\right)$, with the MSD results presented in Fig. 52 for varying $\gamma$ values. ?

Physically, when a dimer diffuses only a fraction of its size from the initial location it does not yet feel any hindrance by collisions with the neighboring crowders and, thus, moves with a constant temperature-dependent velocity, see Eq. (G). This yields the quadratic growth of the MSD [see Eq. ([6) and Eq. (16) below] and of the mean TAMSD at short times, as shown in Figs. 2 and 52. As physically expected, as the friction coefficient increases and corresponding the relaxation times for translational and rotational motion become shorter, the region of ballistic MSD growth gets limited to shorter times, see Fig. [52.

At intermediate lag times, a crossover behavior of the TAMSD in the crowded solutions of dimers is observed (see Fig. 2) and the computed mean TAMSDs curves start to split for different crowding fractions $\phi$. This regime is realized in the region of lag times $\Delta \approx$ $0.1 \ldots 10 \times \delta \tau$. At the end of this interval, the translational and rotational TAMSDs grow almost linearly with lag time. This ballistic-to-linear TAMSD crossover depends on $\phi$ and is characterized by the first-collision time (see Sec. एШB for the analysis of scaling exponents).

Finally, at very long times, both TAMSDs grow linearly with $\Delta$ and follow BM (2) with $\phi$-dependent effective diffusivities (see Sec. एWव below).

When comparing the behaviors at $\gamma=1$ we observe that for small crowding fractions $\phi$ the MSDs of both translational and angular motions are almost equivalent in the entire range of times to the theoretically expected results for $\left\langle x^{2}(t)\right\rangle$ and $\left\langle\Theta^{2}(t)\right\rangle$. Indeed, for the MSD of a single dimer obeying a potential-free version of Eq. (प0) one expects the MSD growth ${ }^{[15,, 16,, 145,152}$

$$
\left\langle x^{2}(t)\right\rangle \approx 2 D_{x} t\left[1-\frac{\tau_{0}}{t}\left(1-e^{-t / \tau_{0}}\right)\right],
$$

also known in polymer physics for the average extension of a fluctuating worm-like chain (see $\S 127$ in Ref. ${ }^{1533}$ ). For the rotational MSD $\left\langle\Theta^{2}(t)\right\rangle$ we use Eq. (16) with the friction coefficient extracted from the diffusivity results of computer simulations based on relation (3), while the particle mass is now being exchanged with the moment of inertia of the dimer (computed with the potentialequilibrated monomer-monomer separation $r_{0}=1.5 \sigma$ using the known mass of the monomer disks). This gives

\footnotetext{
INote that for the rotational diffusion the cumulative angle is computed in the simulations and, thus, the angle difference is not bounded by $2 \pi$. Therefore, for $n$ full rotations of a dumbbell in units of panel (B) of Fig. 2 the azimuthal MSD amounts to $(2 \pi n)^{2}$.
}

the characteristic time of rotational relaxation of the motion, denoted $\tau_{0, \Theta}$. The diffusion of dimers is anisotropic with respect to their axes at short times, when $t \ll \tau_{0, \Theta}$, and it turns isotropic at long times, when $t \gg \tau_{0, \Theta}$.

The crossover from the short-time ballistic to the longtime linear behavior of the rotational and translational MSD and mean TAMSD takes place at times $\sim \tau_{0}$ and $\sim \tau_{0, \Theta}$, respectively, for weakly crowded systems and does so monotonically in terms of reduction of the timelocal scaling exponent, see Eq. (I7]) below. In contrast, the crossover emerges at considerably earlier times for highly crowded solutions of the dumbbells, see Figs. 2 and $\mathrm{B}$, accompanied by a nonmonotonic variation of the respective exponents $\beta_{x}$ and $\beta_{r}$. At high crowding fractions at intermediate times - where the subdiffusive behavior is most pronounced, see also Fig. B-the theoretically expected and observed MSD are rather disparate in magnitude, see Fig. \$3.

We emphasize that for star-shaped crowders a similar-but even more pronounced-non-monotonicity in the variation of these scaling exponents was detected at intermediate lag times, i.e., in the crossover region from the ballistic to the linear diffusion regime, see Fig. $6 \mathrm{~B}$ in Refs. ${ }^{93,94}$. I We find an equivalence of the ensembleand time-based averaging for translational and rotational motion of the dimers. Namely, both in the short- and long-time regimes, we observe $\left\langle\overline{\delta_{x}^{2}(\Delta)}\right\rangle \approx\left\langle x^{2}(\Delta)\right\rangle$ and $\left\langle\overline{\delta_{r}^{2}(\Delta)}\right\rangle \approx\left\langle\Theta^{2}(\Delta)\right\rangle$ indicative of ergodic behavior in this Boltzmann-Khinchin sense $\mathrm{e}^{\sqrt{24}, 25}$, see Fig. \$4. This observation agrees with the results for the diffusion in crowded solutions of star-shaped crowders examined in Refs. ${ }^{93,194}$ (see also Sec. $\square \nabla B$ below). We emphasize also an extremely small dispersion of individual TAMSDs around their mean, both for conditions of weak crowding (expected ${ }^{[156}$ for massive-BM motion ${ }^{[15,, 166}$ ) and heavy crowding, see the results in Fig. 54. This fact thus confirms the ergodicity of self-diffusion of dumbbells in crowded dispersions also in terms of the reproducibility of the TAMSD realizations $\sqrt{24, \sqrt[25]{25}}$, for both translation and rotational motion. ${ }^{* *}$

"Note that a nonmonotonic variation of the TAMSD scaling exponent with lag time was also detected, e.g., for anomalous diffusion of proteins and lipids on/in lipid membranes ${ }^{130,131}$ and in the model of tracer diffusion in driven lattice Lorentz gases of immobile obstacles $1.54,1.5 .5$.

** We do not quantify here the spread of individual TAMSDs and the respective dispersion determining the value of the ergodicity

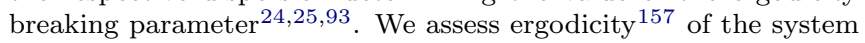
solely from the equivalence of the MSD and the mean TAMSD at short lag times. 

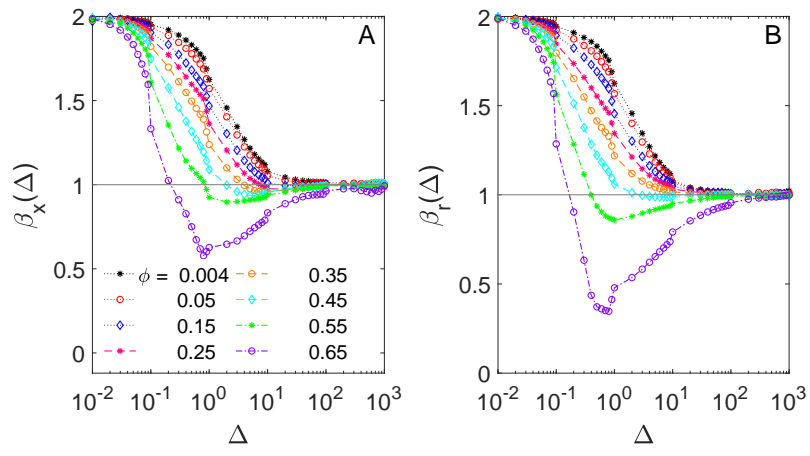

FIG. 3: Time-local scaling exponents of (A) translational and (B) rotational TAMSDs ([17) computed from the simulation data of Fig. $\square$ for varying crowding fractions $\phi$ of the dimers.
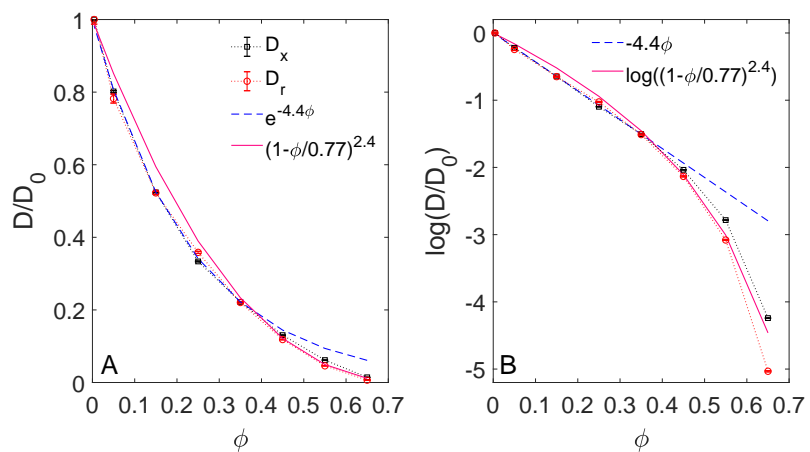

FIG. 4: (A) Long-time translational and rotational diffusivities as functions of the crowding fraction $\phi$, shown together with a fit to an exponential decay (एप.) for small $\phi$ and a quadratic fit (20) for large $\phi$ (see the legend). The values are normalized with respect to $D_{0}=D(\phi=0.004)$ corresponding to a single dimer in the simulation box, see Tab. [1. (B) $\log _{e}$-scale representation of the simulation data from panel (A), with the same asymptotes shown.

\section{B. Time-local scaling exponents}

To examine the $\phi$-dependence of the TAMSD in more detail, the time-local scaling exponents $\beta_{x}$ and $\beta_{r}$ are evaluated as ${ }^{1.58}$

$$
\beta_{\{x, r\}}(\Delta)=\frac{\mathrm{d} \log \left(\left\langle\overline{\delta_{\{x, r\}}(\Delta)^{2}}\right\rangle\right)}{\mathrm{d} \log (\Delta)} .
$$

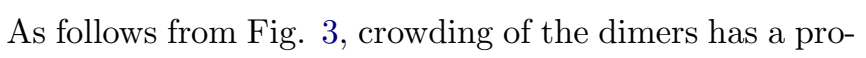
found impact on the behaviour of the scaling exponents at intermediate lag times, $\Delta \approx 0.1 \ldots 10 \times \delta \tau$. Namely, at very short times both scaling exponents $\beta_{x}$ and $\beta_{r}$ equal two for all crowding fractions of the dimers (the ballistic regime, see Eq. (河)), while for long lag times they both expectedly approach unity. In a dilute system, the crossover from the ballistic to the linear growth of the TAMSD occurs similarly to that for the MSD of a massive BM particle ${ }^{\sqrt{15}}$, see also Eq. (16). Namely, the scaling exponent decreases monotonically from $\beta_{\{x, r\}}=2$ at short to $\beta_{\{x, r\}}=1$ at long times, see Fig. 52.

The translational and rotational motion of the dimers are correlated in terms of variations of their scaling exponents. We observe distinct subdiffusive $\beta_{x}$ and $\beta_{r}$ for the most crowded conditions, with the subdiffusion being more pronounced for rotational than for translational motion, compare the values of the dips at $\phi=0.65$ in Figs. 3A and 3B. These reduced values of $\beta_{r}$ at intermediate lag times and at high $\phi$ values are physically connected with a stronger impediment of rotational motion of a dimer by its neighbors, as compared to restrictions on possible translational motion of the same dumbbell. $\dagger \dagger$

We refer the reader to Figs. 21 and 22 in Ref. ${ }^{106}$ for the analysis of $\left\langle x^{2}(t)\right\rangle$ and $\left\langle\Theta^{2}(t)\right\rangle$ in highly crowded 2D solutions of active dumbbells. This system also reveals nonmonotonic variations of the scaling exponents in the transitional regime after the initial ballistic growth, with more pronounced subdiffusion for rotations for $\phi \gtrsim 0.6$. †

\section{Diffusion coefficients}

In the long-time limit, the exponents $\beta_{x}$ and $\beta_{r}$ converge to unity for all $\phi$ values, Fig. 3. Therefore, the $\phi$ dependence of the mean TAMSDs $\left\langle\overline{\delta_{x}^{2}(\Delta)}\right\rangle$ and $\left\langle\overline{\delta_{r}^{2}(\Delta)}\right\rangle$ at long times stems solely from the $\phi$-dependence of the respective translational $D_{x}$ and rotational $D_{r}$ diffusivi-

${ }^{\dagger}$ Note that variation of the moment of inertia of the dumbbelle.g., via altering its mass distribution and size- one can tune the rotational relaxation time $\tau_{0, \Theta}$. Coincidentally, for the currently chosen model parameters the regions of the TAMSD-based subdiffusion for translational and rotational motions are observed at nearly the same lag times, see panels (A) and (B) of Fig. B. Simulating dimers with the same geometric shape and total mass but with significantly different moment of inertia $I$-positioning, e.g., artificially the whole mass in the central point of the dimer and thus making $I=0$ - could also be performed. This would answer the question regarding coupling and inter-relation of the diffusion properties (the region of subdiffusion, values of the scaling exponents, etc.) for translational and rotational motion of crowded solutions stemming from the inertia of the dumbbells versus from correlations in their motion.

$\ddagger \ddagger$ Note that the diffusion of $2 \mathrm{D}$ dimers has recently also been considered experimentally and theoretically in Ref. ${ }^{102}$, with the focus on translational-rotational coupling. Specifically, at low $\phi \approx 0.14$ realized in experiments ${ }^{[02}$ the diffusion of nearly independent dimers was assumed. In contrast, our dumbbells are often at high $\phi$, when the assumption of "independence" is violated. In addition to obtaining the low-order moments, a generalized scattering function was derived ${ }^{102}$. It was found that translational-rotational coupling is caused by anisotropic diffusion of the dimers parallel and perpendicular to their axis, also detected in granular gases $\frac{123}{2}$. While we observe a similar coupling of motion, see Fig. B, no longitudinal and transversal diffusivities of the dumbbells were recorded in current computer simulations. 
ties, evaluated in this regime of long-time BM as

$$
D_{\{x, r\}}(\Delta)=\left\langle\overline{\delta_{\{x, r\}}(\Delta)^{2}}\right\rangle /(2 \Delta) .
$$

The fits of the $\operatorname{TAMSD}(\Delta)$ are executed at lag times in the region $\Delta=10^{2} \ldots 10^{4} \times \delta \tau$. The normalized diffusivities $D_{x}$ and $D_{r}$ as functions of varying crowding fractions $\phi$ are found to display similar behaviors. At small $\phi$, both translational and rotational diffusivities decay exponentially,

$$
D_{\{x, r\}}(\phi) \propto e^{-\phi / \phi_{\exp }},
$$

as evidenced in Fig. $\mathbb{B B}$.

The law ([1.9) at small crowding fractions is also consistent with the linear-in- $\phi$ decrease of the diffusivity predicted theoretically and observed experimentally for concentrated suspensions of spheres in $3 \mathrm{D}, D(\phi) \approx 1-$ $\phi / \phi_{\text {exp }}$, see, e.g., Refs. ${ }^{159,1160}$ and also Ref. ${ }^{103,1617}$. A (slightly compressed) exponential decay of the tracer diffusivity in various hydrogel-like polymeric networks due to effects of steric obstruction was found and rationalized recently in Ref. $\$ 62$.

For large $\phi$, we find a power-law dependence on the crowding fraction, see Fig. [4B. Similar dependencies were detected, e.g., for a system of polydisperse hard

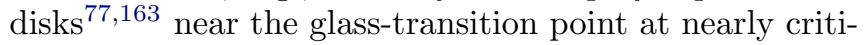
cal fractions of the crowding particles $\phi^{*}$, namely

$$
D_{\{x, r\}}(\phi) \propto\left(\phi-\phi^{*}\right)^{2.4} .
$$

Similar values of the "critical exponent" were proposed for tracer self-diffusion in 3D hard-sphere suspensions, where $D(\phi) \propto\left(\phi-\phi^{*}\right)^{2}$, see Refs. ${ }^{[64,165}$. The dependence (20) is also in agreement with the results of modecoupling theory the translational and rotational diffusivities are affected by varying $\phi$ values to a similar extent, in part because motions of disks imply also changes in orientation of the dimer (and, thus, changes in the angle $\Theta(t)$ ). Of course, the relatively small scaling window available only allows for a relatively qualitative analysis.

This fact contrasts to some extent the properties of diffusion in the crowded solutions of three-arm stars studied in Refs. ${ }^{03,94}$, where the $D(\phi) \propto\left(\phi-\phi^{*}\right)^{2 \ldots 2.4}$ dependence was also detected. Namely, the translational selfdiffusion of stars was found reduced more pronouncedly in crowded conditions, as compared to their rotational diffusion, see Fig. 7 of Ref. ${ }^{0.3}$. This is a physically expected trend because of a more isotropic overall appearance of the star-shaped crowders, as compared to the elongated, ellipsoidal-like shape of the dumbbells here. The translational motion for the stars and rotational motion of the dumbbells are, therefore, expected to be diminished stronger at a fixed high fraction of crowding.

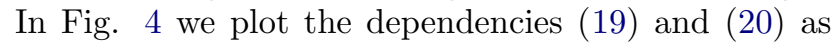
the best fits in their respective ranges of fractions $\phi$, realized for the parameters $1 / \phi_{\exp } \approx 4.4$ and $\phi^{*} \approx 0.77$ for our computer simulation data (see the legend of Fig. 四).
The crossover between these two apparent regimes - an exponential decay ([Q9) in absence of subdiffusion (viscous) and a roughly quadratic dependence (201) for subdiffusion (viscoelastic) - takes place at $\phi \approx 0.35 \ldots 0.45$. Therefore, it occurs in the same range where the subdiffusive behavior for the TAMSD starts to emerge, see Fig. 3. We emphasize that - in contrast to the stronger subdiffusion for rotational motion of the dimers under conditions of moderate-to-severe crowding (see Fig. B)the translational and rotational diffusion coefficients are affected by self-crowding to a nearly the same degree, see Fig. 目.

We refer the reader to the studies of concentrated dispersions of (identical) spheres and of colloidal suspen-

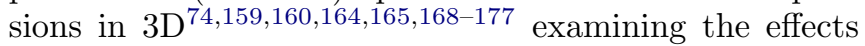
of hydrodynamic interactions. In a number of these studies, the dependence of the long-time self-diffusion coefficient of the spheres [with hydrodynamics] was proposed to have a quadratic form,

$$
D(\phi) \propto(\phi-\bar{\phi})^{2}
$$

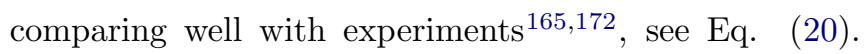
Hydrodynamic effects were considered in detail also in Refs. ${ }^{65,1776}$; we also refer to the recent comparative analysis $\frac{177}{177}$ and to Refs. $\frac{161,175,, 178-180}{180}$ for the examination of translational and rotational diffusion in dense suspensions of charged colloids, see also Ref. ${ }^{176}$. A detailed experimental study of self-diffusion in concentrated solutions of bovine serum albumin proteins -including an in-depth discussion of the $D(\phi)$-dependence in the presence of hydrodynamic and electrostatic interactions - can be found in Ref. ${ }^{170}$.

\section{Dimer-displacements PDF}

The PDFs of translational $p\left(\delta_{x}\right)$ and rotational $p\left(\delta_{r}\right)$ displacements of dumbbells, computed in the range $\Delta=$ $0.1 \ldots 80 \times \delta \tau$, are plotted in Fig. 5 for the most crowded system at $\phi=0.65$. At short lag times, for $\Delta=0.1 \times \delta \tau$, the PDFs for both types of motion expectedly have Gaussian-like profiles. At longer times, the translational PDF remains nearly Gaussian, while the rotational PDF becomes nearly Laplacian (e.g., exponential, as quantified in Sec. एWE below). This feature is visible from the nearly straight tails of the PDF in log-linear scale, as presented in Fig. 5B. This fact indicates that the rotations of dumbbells on large azimuthal angles are much more frequent than the expectation from a Gaussian form of the rotational PDF. We stress here that the statistical observables examined for the crowded solutions of

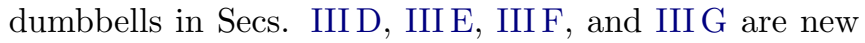
compared to the analysis of self-diffusion of star-shaped crowders performed in Refs. ${ }^{93,94}$. 

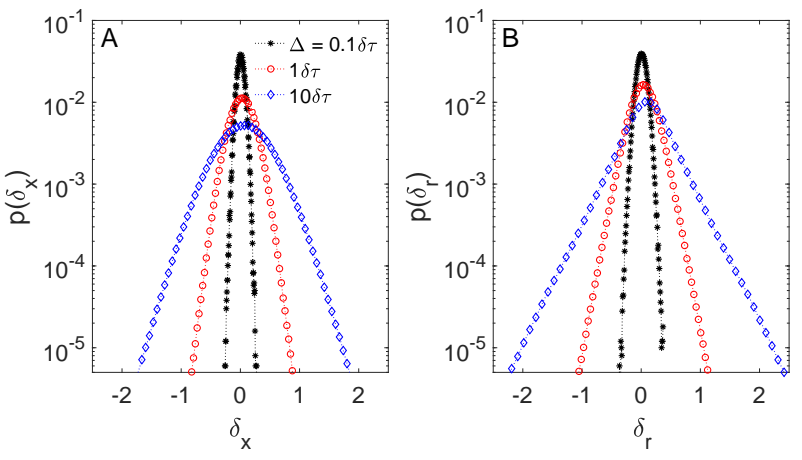

FIG. 5: (A) Translational and (B) rotational displacementPDFs for the highest crowding fraction $\phi=0.65$, computed for several values of the lag time $\Delta$ (see the legend) and for other parameters (such as $m, \gamma, \tau_{0}, \epsilon$, and $T$ ) as in Fig. (2.

\section{E. Non-Gaussianity parameter}

The degree of non-Gaussianity of the PDF shapes of both translational and rotational displacements of the dumbbells is quantified in terms of the non-Gaussianity parameter $G^{[27}$. It is related to the kurtosis (denoted as Kurt below) of the PDF distributions of the displacements of the dimers as

$$
\begin{aligned}
& G_{\{x, r\}}(\Delta)=\operatorname{Kurt}\left[p\left(\delta_{\{x, r\}}(\Delta)\right)\right] / 3-1 \\
& =\frac{1}{3} \frac{\left\langle\left[\delta_{\{x, r\}}(\Delta)-\left\langle\delta_{\{x, r\}}(\Delta)\right\rangle\right]^{4}\right\rangle}{\left(\left\langle\delta_{\{x, r\}}^{2}(\Delta)\right\rangle-\left\langle\delta_{\{x, r\}}(\Delta)\right\rangle^{2}\right)^{2}}-1 .
\end{aligned}
$$

Here, the $n$th moment of the PDF of the displacements $p\left(\delta_{\{x, r\}}\right)$-denoted as $\left\langle\delta_{\{x, r\}}^{n}\right\rangle$ below-is defined for natural numbers $n \geq 1$ as

$$
\left\langle\delta_{\{x, r\}}^{n}\right\rangle=\int_{\infty}^{\infty} \delta_{\{x, r\}}^{n} p\left(\delta_{\{x, r\}}\right) \mathrm{d} \delta_{\{x, r\}} .
$$

Thus, in expressions (221) and (2:3) the ensemble-averaged moments are used, as compared to the TAMSDs given by Eqs. (14) and (15).

In Fig. 6 we present the lag-time dependencies of the non-Gaussianity parameters (22) for varying crowding fractions $\phi$. Corroborating the Gaussian PDFs at short times (see Fig. 5]) the values of $G_{\{x, r\}}$ are comparatively small in this regime. The values of $G_{x}$ and $G_{r}$ increase and reach a maximum at intermediate lag times. At long lag times, extending to the regime of BM for the respective MSD and TAMSD, the values of $G_{x}$ and $G_{r}$ tend to relatively small values again. These nearly Gaussian PDFs for the displacement distributions obtained from our simulations at very long times - in the BM-regime of the MSD at $\Delta=80 \times \delta \tau$, as shown in Fig. 6-are also expected theoretically from the central limit theorem. Note here that the longest time $\Delta=10 \times \delta \tau$ for the computed
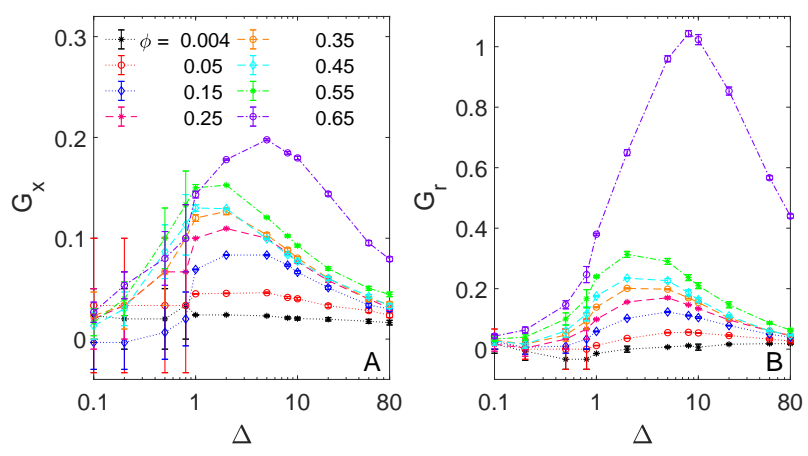

FIG. 6: Non-Gaussianity parameter (22) for (A) translational and $(\mathrm{B})$ rotational motion of the dumbbells plotted against the lag time $\Delta$ for a set of varying $\phi$ (see the legend). The lines connecting the points guide the eye. Note that panels (A) and (B) have different vertical scales.

rotational displacement-PDF in Fig. $5 \mathrm{~B}$ is still considerably shorter than the longest time $\Delta=80 \times \delta \tau$ in the plot of Fig. GB for the respective computed $G_{r}$.

As expected, the most pronounced deviations from Gaussianity are detected for the displacement distributions $p\left(\delta_{\{x, r\}}\right)$ at the maximal crowding fraction, $\phi=$ 0.65 . The maximum of the translational non-Gaussianity parameter is $G_{x} \approx 0.2$, while the maximum of the rotational non-Gaussianity parameter is significantly larger, $G_{r} \approx 1$. The latter fact indicates again a nearly Laplacian shape of the rotational-displacements PDF illustrated in Fig. [BB. $\S \S$ We also demonstrate in Fig. 5.5 that the second moments for the PDFs $p\left(\delta_{\{x, r\}}(\Delta)\right)$ when computed from the simulation data shown in Fig. 5-reveal similar features in their lag-time dependencies in the range $\Delta=0.1 \ldots 80 \delta \tau$ for varying $\phi$ fractions, as those of the respective TAMSDs $\left\langle\overline{\delta_{\{x, r\}}^{2}(\Delta)}\right\rangle$ shown in Fig. 2A,B.

\section{F. ACF of displacements and rotations}

We now evaluate the velocity-ACF $C_{x}$ for the translational displacements $\delta_{x}$, rotational displacements $\delta_{r}$ and the displacements $\delta_{d}$ of the relative coordinate in the dimers. With the time difference $\tau$ between the two displacements - the correlation time - the ACF is obtained as the time average,

$$
\begin{aligned}
& C_{x}(\tau, \Delta)=\overline{v_{x}(\tau, \Delta) v_{x}(0, \Delta)} \\
& =\frac{1}{T-\Delta-\tau} \int_{0}^{T-\Delta-\tau} \frac{\delta_{x}(t+\tau, \Delta) \delta_{x}(t, \Delta)}{\Delta^{2}} \mathrm{~d} t,
\end{aligned}
$$

$\S \S$ We remind the reader here that $K u r t=3$ for a Gaussian PDF in 1D, while Kurt=6 for a Laplacian distribution. Therefore, Eq. (22) gives for these two cases $G=0$ and $G=1$, respectively. 

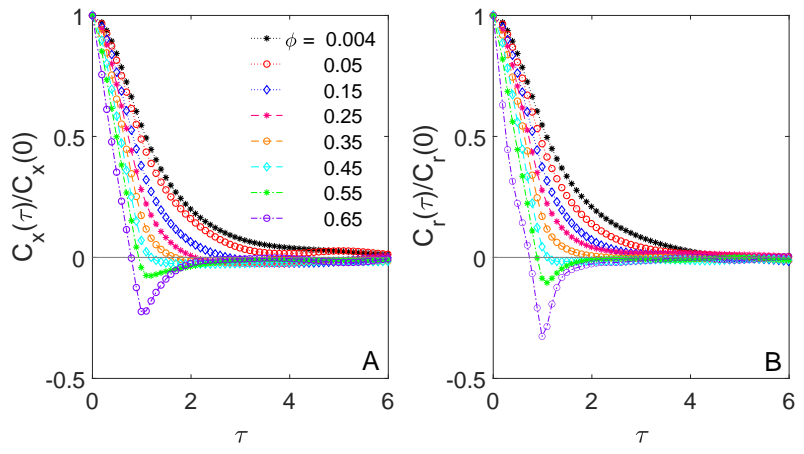

FIG. 7: Normalized (A) translational and (B) rotational displacement ACFs as a function of the correlation time $\tau$ computed for varying crowding fractions $\phi$ (see the legend). The presented ACFs are normalized to the respective initial values at $\tau=0$. Parameters: the lag time in Eq. (24) is $\Delta=1 \times \delta \tau$.

where the factor $1 / \Delta^{2}$ stems from the definition of the "velocity", $v_{x}(t)=[x(\Delta+t)-x(t)] / \Delta \equiv \delta_{x}(t, \Delta) / \Delta$. The dependencies of the time-averaged ACFs for rotational motion and relative-displacement motion of the disks in a dimer, $C_{r}$ and $C_{d}$, respectively, are calculated analogously to expression (24). As the displacements in the integrand of Eq. (24) are shifted by $\tau$, the time averaging is executed along the recorded trajectories up to the trace length $(T-\Delta-\tau)$.

In Fig. 7 , we present the translational and rotational displacement-ACFs plotted against the correlation time $\tau$ for varying $\phi$. For weak-crowding conditions, the decay of the displacement-ACFs $C_{x}$ and $C_{r}$ is monotonic, while for very crowded systems the ACFs display a nonmonotonic behavior. Specifically, initially at $\tau \leq \Delta$ the normalized ACFs start decreasing from unity, reaching a minimum at $\tau=\Delta$. At later stages, for longer correlation times $\tau$, the "recovery" of the ACFs to zero from the region of negative values is detected in the simulation data, see panels (A) and (B) of Fig. 0 for translation and rotational motions, respectively. The displacement-ACFs for larger $\Delta$ values presented in Fig. $\$ 7$ clearly reveal a trend of progressively shallower minima at later $\Delta$. $\boldsymbol{\top}$ बा

These anticorrelations at $\tau=\Delta$ in the highly crowded solutions physically indicate a likely reversal of motion of the dumbbells in the consecutive time step, often referred to as antipersistence. The form of the displacementsACFs $C_{x}$ and $C_{r}$ themselves are reminiscent of those for subdiffusive processes of fractional $\mathrm{BM}$ and of motion governed by the fractional Langevin equation ${ }^{[24-[26,31,322}$.

\footnotetext{
ฯ Note that a similar trend was experimentally detected, e.g., for the diffusion of doxorubicin ${ }^{[181]}$ drug molecules in confined silica nanoslits in Ref. $\frac{[182}{}$, while deeper minima of the velocity-ACFs at later time shifts were found, in contrast, for chromatin dynamics in computer simulations of viscoelastic subdiffusion of chromosomal loci in Ref. ${ }^{\llbracket 83 .}$.
}
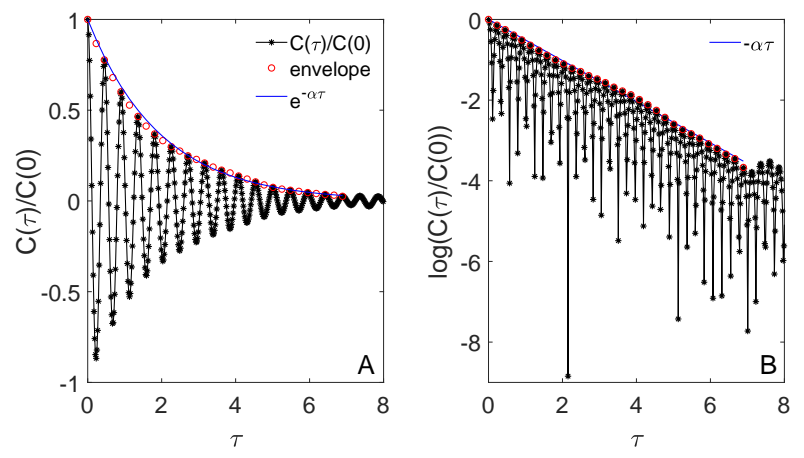

FIG. 8: (A) Normalized displacement ACF $C_{d}$ of the relative coordinate of a single dimer $(\phi=0.004)$ as a function of the correlation time $\tau$. This crowding fraction corresponds to a single dimer in the simulation box, see Tab. II. The decay of the ACF amplitude is fitted by the envelope function $C_{d}^{\text {env }}(\tau)$ given by (25). (B) Data from panel (A) in $\log _{e}$-scale (shown together with a linear asymptote for the same exponential decay).
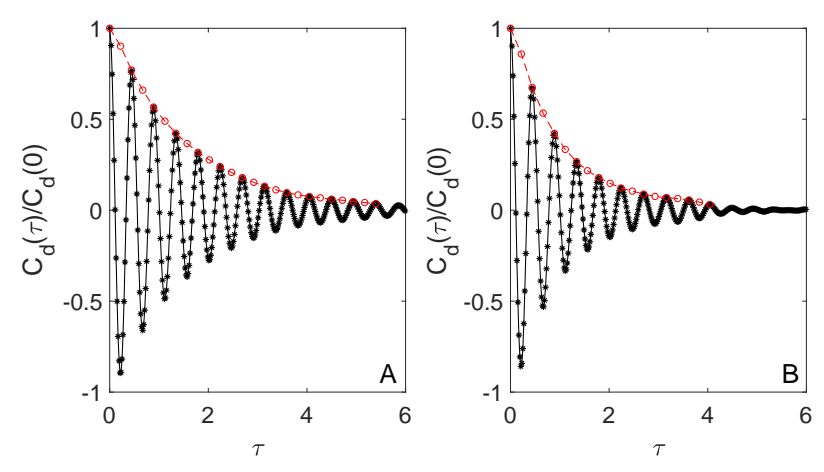

FIG. 9: Displacement ACF $C_{d}$ for (A) $\phi=0.05$ and (B) $\phi=0.15$. The red circles are given by Eq. (25]).

These two models of non-Brownian diffusion are often used for the mathematical description of subdiffusion in crowded and viscoelastic media ${ }^{26,1,184}$. ${ }^{* * *}$

\section{G. Relative-coordinate ACF}

The normalized $\mathrm{ACF} C_{d}(\tau) / C_{d}(0)$ for displacements of the relative coordinate - defined in the time-averaged sense as in Eq. (24) - is plotted versus $\tau$ for $\phi=0.004$ (a single dimer in the simulation box) in Fig. \$. The oscillations of $C_{d}(\tau)$ indicate vibrational "breathing modes"

** Note that the direct comparison of the computed ACFs from simulations with those of, for instance, fractional Brownian motion with a constant Hurst exponent $H$ is not straightforward due to the fact that the time-local anomalous-diffusion exponents in the simulations vary strongly with lag time, see Fig. 3 . 

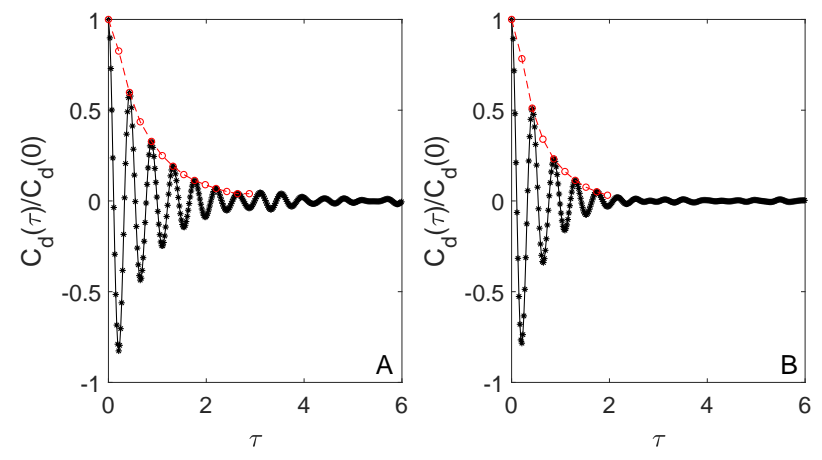

FIG. 10: The same as in Fig. 9, but computed for (A) $\phi=$ 0.25 and (B) $\phi=0.35$. The red circles are given by Eq. (2.5I).
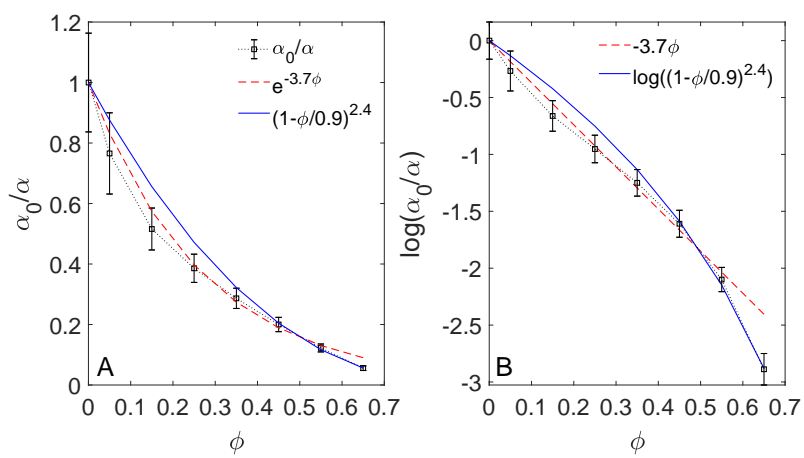

FIG. 11: Normalized inverse decay constant of $C_{d}^{\text {env }}(\tau)$ given by $\alpha_{0} / \alpha$ from Eq. (2.5) shown as function of crowding fraction $\phi$ in linear (A) and $\log _{e}(\mathrm{~B})$ scale. The fit functions used are listed in the legends. Here, $\alpha_{0}=\alpha(\phi=0.004)$ and the error bars are indicated.

of the dimers and the exponentially decaying amplitude of these oscillations is due to the damping effects of the environment. As effective damping increases with $\phi$, the oscillations become suppressed and their amplitudes decay faster with time as $\phi$ grows, compare the results of Fig. 8 for a single dimer to those of Figs. 9 and 10 obtained for crowding fractions $\phi=\{0.05,0.15,0.25,0.35\}$.

We compare these oscillations of the dumbbells to those of a noise-driven damped harmonic oscillator (see $\S 25$ of Ref. $\left.{ }^{[85}\right)$. The period of $C_{d}(\tau)$ oscillations, denoted by $\mathrm{T}$, is found to be only moderately dependent on $\phi$, see Fig. \$8A. For the frequency of dimer oscillations, $\omega=2 \pi / \mathrm{T}$, for weak crowding [and no effects of the neighbors] we find an excellent agreement with the eigenfrequency of the damped harmonic oscillator ${ }^{16,}, 18.5$ $\omega_{0}=2 \mathrm{k} / \mathrm{m}$ [for free oscillations, with the reduced mass $m / 2]$.

With increasing $\phi$ the simulation data predict increasing frequency of $C_{d}(\tau)$ oscillations. Physically, this trend is expected because - for a given energy "influx" into the system due to fluctuations of the thermal bath - the reduced space available for expansion of the dumbbells in progressively more crowded solutions gives rise to oscilla- tions at higher frequencies. The frequency of the damped oscillator, however, has the opposite trend with increasing crowding fraction [if we assume that $\alpha(\phi) \propto \gamma(\phi)$, as in Fig. $58 \mathrm{~B}]$. In both cases, however, rather insignificant $\omega(\phi)$ variations are found, as quantified in Fig. $\mathbf{5 8}$.

The $\phi$-dependence of the decay rate $\alpha(\phi)$ of the envelope function $C_{d}^{\mathrm{env}}(\tau)$ of the oscillating ACF-function $C_{d}(\tau)$ is determined from the exponential fit

$$
C_{d}^{\mathrm{env}}(\tau) \propto e^{-\alpha(\phi) \times \tau}
$$

as shown in Fig. 11]. To relate $\alpha(\phi)$ to the exponential dependence of $D(\phi)$ in Fig. 目 and Eq. ([.]), the normalized inverse decay constant is shown in Fig. $1 \mathrm{~TB}$. We find that, similarly to $D(\phi)$, the reciprocal constant $1 / \alpha(\phi)$ decays nearly exponentially with $\phi$ and only the most crowded solutions reveal deviations from this behavior. The inverse representation was chosen in Fig. ए1/A in order to compare the resulting dependence of $\alpha_{0} / \alpha(\phi)$ to a decreasing $D(\phi)$ in Fig. WA [because $\alpha(\phi) \propto \gamma(\phi)$ and $\gamma(\phi) \propto 1 / D(\phi)$ in virtue of relation (B)]. The decaying oscillations of the displacement-ACF $C_{d}(\tau)$ of the dumbbells are typical for a damped harmonic oscillator ${ }^{[6,185.5}$. This similarity is expected because Eq. (10) reduces to the Langevin equation of a randomly driven harmonic oscillator when a single dimer is considered. In the dilute regime the Lennard-Jones interactions can in the first order be neglected because the distance between the two monomers is $\sim r_{0}$. In addition, as proposed in Ref. ${ }^{[16}$, it gives a physical meaning to the decay rate $\alpha \sim 1 / \tau_{0}$. Therefore, the vibrations of the dimers for weakly crowded systems can be rationalized by those of randomly driven harmonic oscillators with the crowding-fraction dependent damping constant, $\gamma_{\text {eff }}(\phi)$.

We note that some deviations from the behavior (25) observed at large $\phi$ in Fig. 10 indicate a potential change to another diffusion regime. Due to worsening accuracy of the simulation data for $\alpha$ in this regime, however, the crossover is not clear. This high-density regime may be modeled by a fractional Langevin equation with powerlaw memory kernel in the presence of an external harmonic potential ${ }^{[32,35 .}$. To be confident about these observations significantly more elaborate simulations are needed.

\section{DISCUSSION AND CONCLUSIONS}

\section{A. Summary of the main results}

Based on the results of extensive computer simulations we studied the stochastic dynamics of a crowded system of dimers in two distinct regimes of crowding fractions $\phi$. In the region of small $\phi$, at $\phi \lesssim 0.35$, we observed standard BM of the dimers supported by the behavior of the scaling exponent in Fig. 3 and by variation of the displacement-ACF of the COM of the dumbbells shown in Fig. 7. Note that the deviations of the ACF from 
zero at larger $\tau$ values are due to progressively worsening statistics. In this weak-crowding regime, the effects of crowding can be modeled via an effective constant $\gamma_{\text {eff }}$, compare Figs. 3 and 52. Using Eq. (31), the variation of $\gamma_{\text {eff }}(\phi)$ obtained from Fig. $\mathbb{t}$ is, therefore, also nearly exponential with $\phi$, see expression ([1.9).

At high-crowding conditions, with $\phi \gtrsim 0.35$, we observed subdiffusive scaling exponents (with particularly severe subdiffusion for rotational motion) and the displacement-ACFs displaying anticorrelations indicative of, e.g., viscoelastic-type subdiffusion ${ }^{[24]}$. We found that translational and rotational motion of the dimers are coupled in the region of intermediate lag times where crowding-induced subdiffusion is detected, see Fig. 3 and also Refs. ${ }^{97,102}$. In this regime, with increasing crowding fractions $\phi$ the diffusivity decreases according to a powerlaw form, Eq. (201).

A model that combines subdiffusion and anticorrelations is subdiffusive fractional Brownian motion ${ }^{[186}$ as well as the closely related model of motion governed by a fractional Langevin equation ${ }^{187}$. These two mathematical models of anomalous but ergodic diffusion are often associated with the physical motion of [endogenous and exogenous] tracers in viscoelastic environments ${ }^{24,}, 52,128$, such as those of the cell cytoplasm and of artificially crowded fluids in vitro. Thus, we can interpret the two distinct regimes regarding the observed effects of crowding on the diffusion of the dimers as those of a viscous liquid at low $\phi$ and viscoelastic liquid at high $\phi$ fractions. A concentration-dependent transition between viscous and viscoelastic diffusion was also proposed in Ref. ${ }^{188}$.

\section{B. Physical rationales and further discussion}

We found two distinct diffusion regimes in our crowded system of passively diffusing dumbbells. In dilute systems, we obtained a behavior consistent with standard BM featuring Gaussian displacement-PDFs and nearly exponentially decaying (Laplacian) rotational ACFs. The effect of $\phi$ on the diffusion could be modeled here as effective damping, obtained from the exponential decrease of the diffusivity with $\phi$, see Eq. ([1.). Similarly, the damping of internal vibrations of the dimers was shown to depend exponentially on $\phi$. It was found to give rise to a quicker decay of oscillations in the respective internal-ACF describing relative separations of monomers in a given dumbbell.

For highly crowded systems, we instead observed transient subdiffusion of the dumbbells and the non-Gaussian PDFs of their anticorrelated displacements. In this regime, the average diffusivity of the dumbbells was shown to decrease as a power law with increasing crowding fraction $\phi$, Eq. (20).

We tentatively attribute these two different diffusion regimes found in simulations to a crowding-induced crossover from a purely viscous to a viscoelastic behavior of the diffusion environment. Physically, this viscoelas- ticity at high $\phi$ can stem from shape-responsiveness and "concerted" motions of neighboring dumbbells. Namely, noise-driven "jiggling" of dimers leads to highly frequent collisions and interactions between them. A finite adaptability of elastically responsive dumbbells can thus give rise to correlated shape deformations of the neighbors at high $\phi$ values. A displacement of a dimer COM position from its equilibrium value gets reversed by the elastic environment of the neighbors leading to anticorrelated motions of a given dumbbell, see Fig. 7. To verify this hypothesis, further studies should be done to include a $\phi$-dependence of inter-monomer forces and for different number of neighbors.

Recent Bayesian model-assessment analysis ${ }^{\text {189-191] }}$ as well as modern machine-learning approaches ${ }^{192-198}$ could then determine relative probabilities of possible diffusion models involved. In the end, certain predictions regarding the diffusion characteristics based on physical properties of the environment of crowders might be possible.

We also observed this transition for the diffusion of single non-connected monomers characterized by a crossover of the diffusivity dependencies, see Fig. 56. Here, the transition occurs at somewhat higher $\phi$ values because solely the Lennard-Jones potential is the source of elasticity in this modified system. This suggests that more complex crowders - such as a linear chain of inter-connected monomers ${ }^{97}$ _ should exhibit such a transition from viscous to viscoelastic behavior at smaller $\phi$ fractions, as compared to those for the dumbbells (work in progress).

To discuss the effects of the dumbbell shape on the diffusive characteristics, we highlight now the key differences of the current results to those for the diffusion of star-shaped crowders reported earlier in Ref. ${ }^{9.3}$. For the star-shaped crowders, transient subdiffusion was found to occur for all crowding fractions (up to $\phi=0.55$ ), while only the most crowded dimer-based systems were shown to exhibit such a behavior, see Figs. 2 and 3 . Similarly, the power-law dependence of the diffusivity on the crowding fraction, $D(\phi)$, was observed in Ref. ${ }^{\frac{93}{3}}$ for all $\phi$ values, while for the dimers we observed this dependence only in a high- $\phi$ regime. Moreover, the crowding fraction corresponding to the glass transition value $\phi^{*}$ for the star-shaped crowders ${ }^{93}$ was smaller than that for the dumbbell-shaped crowders examined in this study, $\phi_{\text {star }}^{*} \approx 0.52$ versus $\phi_{\text {dumb }}^{*} \approx 0.77$, respectively. For starshaped crowders ${ }^{93}$, at "intermediate" time scales strong variations of both super- and subdiffusive behaviors were observed $^{94}$, with a more anomalous TAMSD scaling exponent detected for rotational than for translational motion, similarly to the dimers. Moreover, the diffusion of star-shaped crowders was found ergodic for small $\phi$ values $^{93}$.

These deviations can, in part, be due to different structures of the two types of crowders. The inner monomer of a star-like crowder ${ }^{93,94}$ is connected to three other monomers by springs and, thus, its environment is effectively "viscoelastic" independent of $\phi$ value. In contrast, a disk in a dumbbell is connected to only one other 
monomer, allowing for a more "viscous" medium at low $\phi$. The difference of $\phi_{\text {star }}^{*}$ and $\phi_{\text {dumb }}^{*}$ can be explained by a simpler structure of dimers facilitating their denser packing prior to reaching the glass-transition point.

Although an elastic dimer can be considered as a model of shape-asymmetric proteins ${ }^{[132,1199}, 3 \mathrm{D}$ computer simulations with electrostatic interactions and hydrodynamic effects $^{63}$ - instead of $2 \mathrm{D}$ simulations without these two effects - are required to approach the problem of macromolecular crowding as realized in many biological cells. Crowding by shape-elongated molecules can have implications onto the properties of biomolecular reactions ${ }^{66}$ (including protein folding and protein-protein association) as well as of polymer and DNA looping. The diffusion in the crowded solutions of anisotropic proteins in lipid membranes ${ }^{100,1010}$ and the elongated particles (such as short fragments of DNA and rod-like viruses) adsorbed on lipid membranes are also relevant areas and biophysical systems.

Finally, size- and shape-asymmetric particles (charged dumbbells $^{76}$ and neutral molecules) can also build some

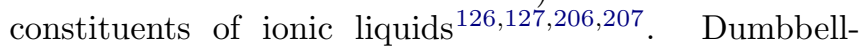
shaped molecules functionalized with ionic liquids were proposed, e.g., as "hybrid" electrolyte for lithium-metal batteries 208 . Soft dumbbell particles with opposite charges $\sqrt{209}-212$ and their applications to dipolar liquids and gels can also be mentioned.

\section{Acknowledgments}

A. G. C. gratefully acknowledges the Humboldt University of Berlin for hospitality and support. The authors thank D. Caetano, S. Kondrat, and R. G. Winkler for correspondence and scientific discussions/comments. R. M. acknowledges financial support by Deutsche Forschungsgemeinschaft (DFG Grant ME 1535/12-1). R. M. thanks the Foundation for Polish Science (Fundacja na rzecz Nauki Polskiej) for support within an Alexander von Humboldt Polish Honorary Research Scholarship.

\section{Abbreviations}

Brownian motion, BM; center of mass, COM; probability-density function, PDF; mean-squared displacement, MSD; time-averaged MSD, TAMSD; autocorrelation function, $\mathrm{ACF}$.

\section{Appendix A: Simulation scheme}

To simulate Eq. (ए0), we use the numerical Verlet velocity algorithm for the positions and velocities of the $i$ th particle. We use it separately for the $x$ - and $y$ component, but present below only the expressions for the $x$-component, namely

$$
\begin{aligned}
& x_{i}(t+\Delta t)=a_{i, x}(t) \Delta t^{2} / 2+v_{i, x}(t) \Delta t+x_{i}(t), \\
& v_{i, x}(t+\Delta t)=\left[a_{i, x}(t)+a_{i, x}(t+\Delta t)\right] \Delta t / 2+v_{i, x}(t) .
\end{aligned}
$$

The integration time-step is $\Delta t=0.005$. For the acceleration of the $i$ th disk, with $x$-component $a_{i, x}(t)$, we use the forces from the potentials (8) and (9) yielding

$$
\begin{aligned}
m a_{i, x}(t) & =\left[-\sum_{\substack{j=1, j \neq i}}^{2 N} \nabla\left(U_{\mathrm{LJ}}\left(r_{i j}\right) \theta\left(2^{1 / 6} \sigma-r_{i j}\right)\right)\right. \\
& \left.-\nabla U_{\text {harm }}\left(r_{i^{\prime} i^{\prime \prime}}(t)\right)\right]_{x} .
\end{aligned}
$$

We describe friction via the $\gamma$-dependent coefficients $c_{\{x 1, x 2\}}(\Delta t)$ and $c_{\{v 1, v 2, v 3\}}(\Delta t)$ given by (see Ref. ${ }^{[150}$ )

$$
\begin{aligned}
& c_{x 1}=\left[\Delta t / \tau_{0}-1-e^{-\Delta t / \tau_{0}}\right] \tau_{0}^{2}, c_{x 2}=\left[1-e^{-\Delta t / \tau_{0}}\right] \tau_{0}, \\
& c_{v 1}=c_{x 2}+\frac{c_{x 2}}{\Delta t / \tau_{0}}+\tau_{0}, c_{v 2}=\frac{c_{x 1}}{\Delta t} ; c_{v 3}=e^{-\Delta t / \tau_{0}},
\end{aligned}
$$

so that

$$
x_{i}(t+\Delta t)=c_{x 1} a_{i, x}(t)+c_{x 2} v_{i, x}(t)+x_{i}(t)+\tilde{\xi}_{x}(t),
$$

$v_{i, x}(t+\Delta t)=c_{v 1} a_{i, x}(t)+c_{v 2} a_{i, x}(t+\Delta t)+c_{v 3} v_{i, x}(t)+\tilde{\xi}_{v_{x}}(t)$

where the noise is modeled by random variables $\tilde{\xi}_{x}(t)$ and $\tilde{\xi}_{v_{x}}(t)$, At $\gamma \rightarrow 0$ - that physically corresponds to the limit of undamped motion and of the Newtonian ${ }^{13.3}$

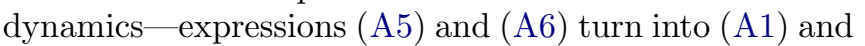
(西2).

\section{Appendix B: Supplementary figures}

* Electronic address: kolja.klett(@)web.de

$\dagger$ Electronic address: a.cherstvy(gmail.com, ht.tns: / /orcid org/(0)(0)-(0)(02-(0516-990()

‡ Electronic address: jaeoh.shin 0 gmail.com, ht.tps: / /orcid.org/00(0)-(00)(02-2:365-5793

$\S$ Electronic address: igor.sokolov(@)physik.hu-berlin.de,

\section{https: / /orcid.org/000()-0(002-4688-9162}

ๆ Electronic address: rmetzler $(a)$ uni-potsdam.de. ht.tns: / /orcid org/(0)(n)-()()(2)-6()1.3-7()20

1 Brown R. A brief account of microscopical observations made in the months of June, July and August 1827, on the particles contained in the pollen of plants; and on the gen- 


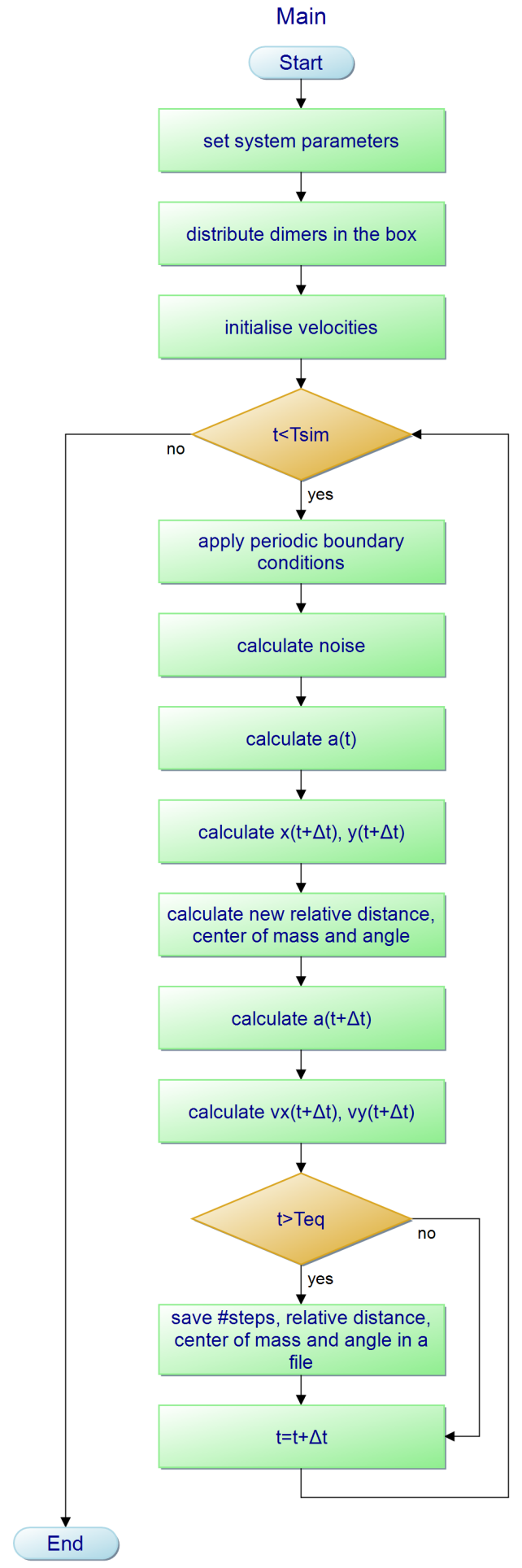

FIG. S1: Flow-chart of computer simulations, with initialization of the system and integration loop.
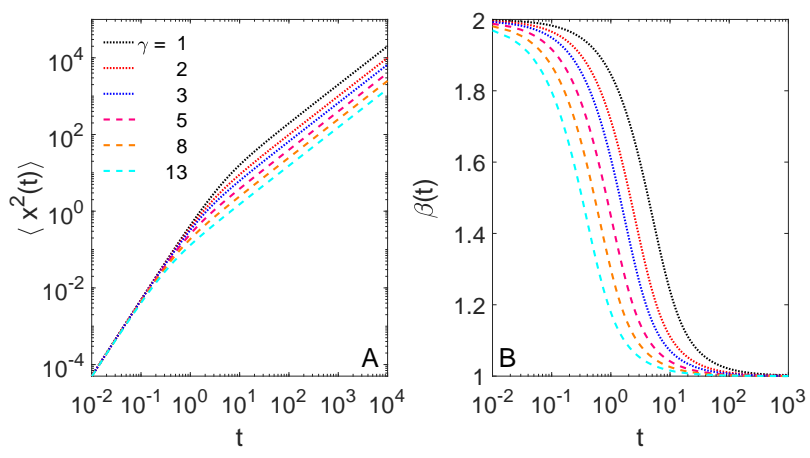

FIG. S2: (A) Translational MSD for the COM motion of a single dimer governed by Eq. (团) (see also Eq. (17) in Ref. ${ }^{[52}$ ) for varying friction coefficient $\gamma$, with $k_{B} \mathcal{T}=1$ and $m=1$. (B) MSD scaling exponent for the simulation data of panel (A), calculated via Eq. ([7).
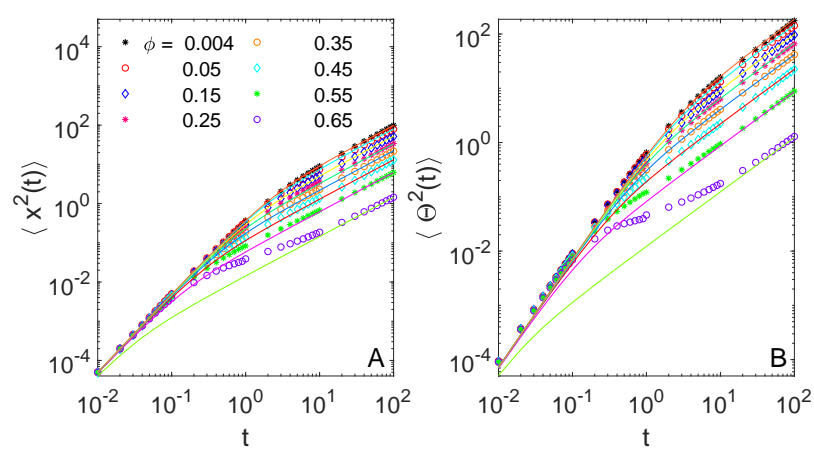

FIG. S3: (A) Translational and (B) rotational MSD for diffusion of dimers at different crowding fractions $\phi$ (see the legend, symbols), plotted for $\gamma=1$ for the TAMSD data of Fig. 2, with the analytical result ([י]) shown as the solid curves.
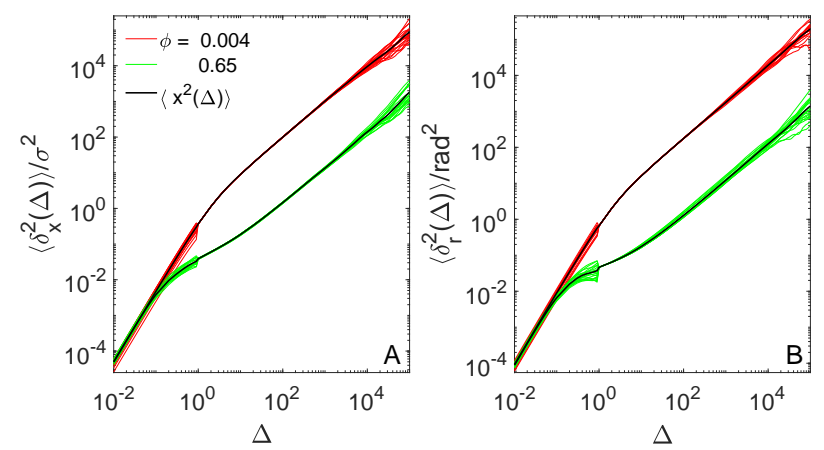

FIG. S4: Direct comparison of the translational (A) and rotational (B) MSDs (the solid black curves) and the TAMSDs (the colored curves) for self-diffusion of dumbbells at two limiting crowding fractions, $\phi_{\min }=0.004$ and $\phi_{\max }=0.65$ (yielding fast and slow diffusion, respectively, see the legend). The results are plotted from the TAMSD data of Fig. 2 and the MSD data of Fig. 53]. Note that, as the integration lag-time interval was splitted into two domains to facilitate the performance, a minor discontinuity at $\Delta=1 \times \delta \tau$ is visible in the data. 

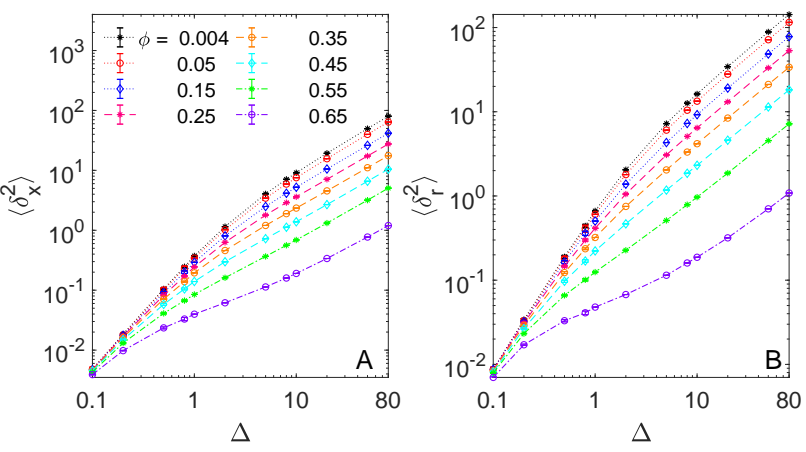

FIG. S5: Second moment of the (A) translational and (B) rotational displacement-PDFs given by Eq. (22:3), presented as a function of lag time $\Delta$ for varying crowding fractions $\phi$.
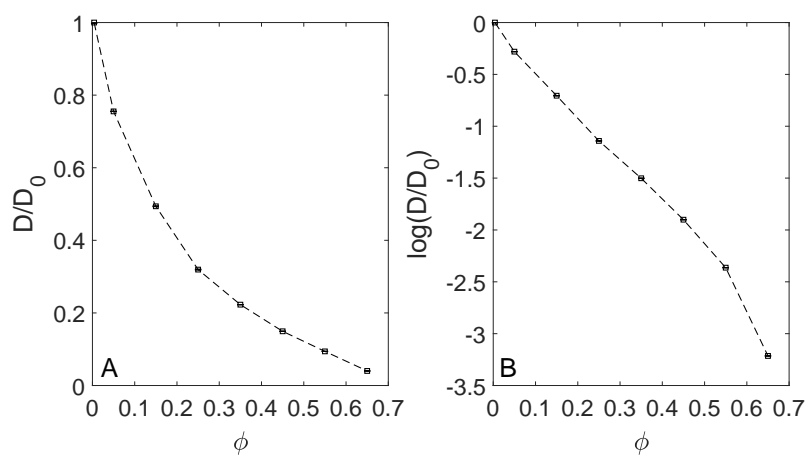

FIG. S6: (A) Translational diffusivity as a function of $\phi$ obtained via expression ([18) from the MSD of diffusion of single, non-connected monomers. (B) Linear- $\log _{e}$ plot of the data of panel (A). Parameters are the same as in Fig. 2.

eral existence of active molecules in organic and inorganic bodies. The Philosophical Magazine. 1828;4(21):161-173.

2 Einstein A. Über die von der molekularkinetischen Theorie der Wärme geforderte Bewegung von in ruhenden Flüssigkeiten suspendierten Teilchen. Ann Physik. 1905;322(8):549-560.

3 Einstein A. Zur Theorie der Brownschen Bewegung. Ann Physik. 1906;19(8):371-381.

4 Einstein A. Theoretische Bemerkungen über die Brownsche Bewegung. Z für Elektrochemie. 1907;13(6):41-48.

${ }^{5}$ Fick A. Über Diffusion. Ann Physik. 1855;170(1):59-86.

6 Bachelier L. Théorie de la spéculation. Annales scientifiques de l'École Normale Supérieure. 1900;3e série, 17:21-86.

7 Sutherland W. LXXV. A dynamical theory of diffusion for non-electrolytes and the molecular mass of albumin. The London, Edinburgh, and Dublin Philosophical Magazine and J Science. 1905;9(54):781-785.

8 Pearson K. The problem of the random walk. Nature. 1905;72:294.

9 von Smoluchowski M. Zur kinetischen Theorie der Brownschen Molekularbewegung und der Suspensionen. Ann Physik. 1906 Jan;326:756-780.

10 Dirac PAM. The physical interpretation of the quan-

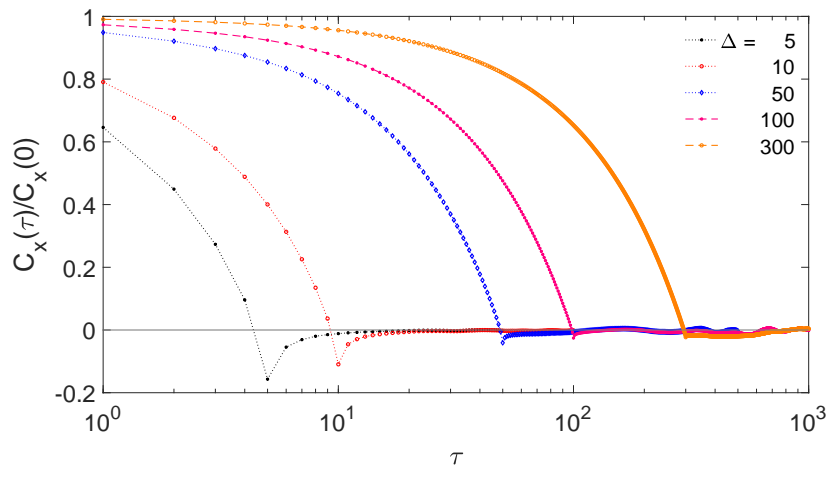

FIG. S7: The same as in Fig. 7, evaluated for the same parameters except for larger values of the lag time, see the legend. All the curves approach unity at vanishing $\tau$ values (the region is not shown in the plot for presentation purposes).
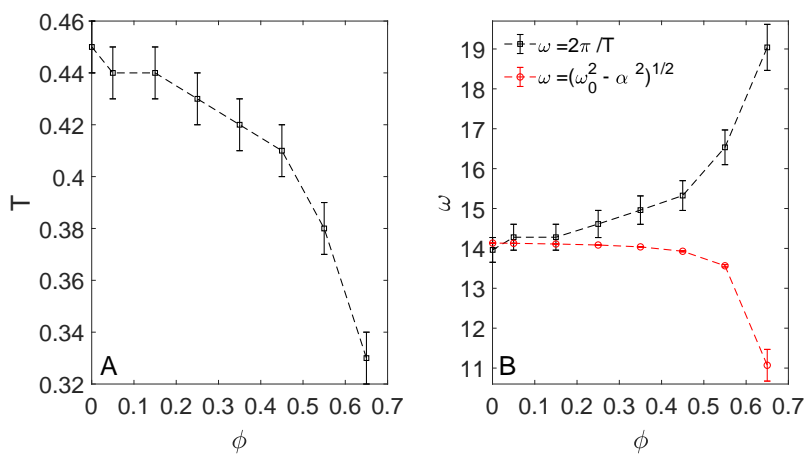

FIG. S8: (A) Variation of the oscillation period $\mathrm{T}(\phi)$ of the ACFs $C_{d}(\tau)$-as obtained from the simulation data of Figs. 8, 9, and 10-versus crowding fraction $\phi$. (B) Comparison of the recalculated frequencies $\omega(\phi)=2 \pi / \mathrm{T}(\phi)$ plotted versus $\phi$ to the frequency of the damped harmonic oscillator ${ }^{[6,,[8.5]}$. The latter is given by $\omega_{\text {osc }}(\phi)=\sqrt{\omega_{0}^{2}-\alpha(\phi)^{2}}$, where the eigenfrequency is $\omega_{0}$ and we assumed $\alpha(\phi) \propto \gamma(\phi) \propto 1 / D(\phi)$.

tum dynamics. Proc Royal Soc London Ser A. 1927;113(765):621-641.

11 Gauß CF. Theoria motus corporum coelestium in sectionibus conicis solem ambientium. Hamburgi: sumtibus Frid. Perthes et I. H. Besser; 1809.

${ }^{12}$ Langevin P. Sur la théorie du mouvement brownien. Comptes-rendus de l'Académie des sciences. 1908;(146):530-533.

13 Newton I. Philosophiae Naturalis Principia Mathematica ("Mathematical Principles of Natural Philosophy"). London; 1687.

14 Stokes GG. On the effect of the internal friction of fluids on the motion of pendulums. Transac Cambridge Phil Soc. 1851;9:8.

15 Uhlenbeck GE, Ornstein LS. On the theory of the Brownian motion. Phys Rev. 1930 Sep;36:823-841.

16 Wang MC, Uhlenbeck GE. On the theory of the Brownian motion II. Rev Mod Phys. 1945;17:323-342.

17 Chandrasekhar S. Stochastic problems in physics and astronomy. Rev Mod Phys. 1943 Jan;15:1-89. 
18 Perrin, Francis. Mouvement brownien d'un ellipsoide - I. Dispersion diélectrique pour des molécules ellipsoidales. J Phys Radium. 1934;5(10):497-511.

19 Perrin, Francis. Mouvement Brownien d'un ellipsoide (II). Rotation libre et dépolarisation des fluorescences. Translation et diffusion de molécules ellipsoidales. J Phys Radium. 1936;7(1):1-11.

20 Bouchaud JP, Georges A. Anomalous diffusion in disordered media: Statistical mechanisms, models and physical applications. Phys Rep. 1990;195(4):127-293.

21 Metzler R, Klafter J. The random walk's guide to anomalous diffusion: a fractional dynamics approach. Phys Rep. 2000;339(1):1-77.

22 Zaslavsky GM. Chaos, fractional kinetics, and anomalous transport. Phys Rep. 2002;371(6):461-580.

23 Metzler R, Klafter J. The restaurant at the end of the random walk: recent developments in the description of anomalous transport by fractional dynamics. J Phys A. 2004 jul;37(31):R161-R208.

${ }^{24}$ Metzler R, Jeon JH, Cherstvy AG, Barkai E. Anomalous diffusion models and their properties: non-stationarity, non-ergodicity, and ageing at the centenary of single particle tracking. Phys Chem Chem Phys. 2014;16:2412824164.

25 Burov S, Jeon JH, Metzler R, Barkai E. Single particle tracking in systems showing anomalous diffusion: the role of weak ergodicity breaking. Phys Chem Chem Phys. 2011;13:1800-1812.

26 Sokolov IM. Models of anomalous diffusion in crowded environments. Soft Matter. 2012;8:9043-9052.

27 Höfling F, Franosch T. Anomalous transport in the crowded world of biological cells. Rep Progr Phys. 2013 mar;76(4):046602.

28 Meroz Y, Sokolov IM. A toolbox for determining subdiffusive mechanisms. Phys Rep. 2015;573:1-29.

29 Metzler R, Jeon JH, Cherstvy AG. Non-Brownian diffusion in lipid membranes: Experiments and simulations. Biochim Biophys Acta (BBA) - Biomembranes. 2016;1858(10):2451-2467.

30 Mandelbrot BB, van Ness JW. Fractional Brownian motions, fractional noises and applications. SIAM Rev. 1968;10(4):422-437.

31 Jeon JH, Metzler R. Fractional Brownian motion and motion governed by the fractional Langevin equation in confined geometries. Phys Rev E. 2010 Feb;81:021103.

32 Jeon JH, Metzler R. Inequivalence of time and ensemble averages in ergodic systems: Exponential versus power-law relaxation in confinement. Phys Rev E. 2012 Feb;85:021147.

33 Wang W, Cherstvy AG, Kantz H, Metzler R, Sokolov IM. Time averaging and emerging nonergodicity upon resetting of fractional Brownian motion and heterogeneous diffusion processes. Phys Rev E. 2021;104:024105.

${ }^{34}$ Cherstvy AG, Wang W, Metzler R, Sokolov IM. Inertia triggers nonergodicity of fractional Brownian motion. Phys Rev E. 2021 Aug;104:024115.

35 Sandev T, Metzler R, Tomovski Z. Correlation functions for the fractional generalized Langevin equation in the presence of internal and external noise. J Math Phys. 2014;55(2):023301.

36 Ślezak J, Metzler R, Magdziarz M. Superstatistical generalised Langevin equation: non-Gaussian viscoelastic anomalous diffusion. New J Phys. 2018 feb;20(2):023026.
37 Vojta T, Skinner S, Metzler R. Probability density of the fractional Langevin equation with reflecting walls. Phys Rev E. 2019 Oct;100:042142.

38 Jeon JH, Chechkin AV, Metzler R. Scaled Brownian motion: a paradoxical process with a time dependent diffusivity for the description of anomalous diffusion. Phys Chem Chem Phys. 2014;16:15811-15817.

39 Thiel F, Sokolov IM. Scaled Brownian motion as a meanfield model for continuous-time random walks. Phys Rev E. 2014 Jan;89:012115.

40 Safdari H, Cherstvy AG, Chechkin AV, Bodrova A, Metzler R. Aging underdamped scaled Brownian motion: Ensemble- and time-averaged particle displacements, nonergodicity, and the failure of the overdamping approximation. Phys Rev E. 2017 Jan;95:012120.

${ }^{41}$ Cherstvy AG, Safdari H, Metzler R. Anomalous diffusion, nonergodicity, and ageing for exponentially and logarithmically time-dependent diffusivity: striking differences for massive versus massless particles. J Phys D. 2021 feb;54(19):195401.

42 Sokolov IM, Heinsalu E, Hänggi P, Goychuk I. Universal fluctuations in subdiffusive transport. EPL (Europhysics Letters). 2009;86(3):30009.

43 Thiel F, Sokolov IM. Time averages in continuous-time random walks. Phys Rev E. 2017 Feb;95:022108.

${ }^{44}$ Kutner R, Masoliver J. The continuous time random walk, still trendy: fifty-year history, state of art and outlook. Eur Phys J B. 2017 March;90:50.

45 Kindermann F, Dechant A, Hohmann M, Lausch T, Mayer D, Schmidt F, et al. Nonergodic diffusion of single atoms in a periodic potential. Nature Phys. 2017 October;13:137-141.

${ }^{46}$ Hou R, Cherstvy AG, Metzler R, Akimoto T. Biased continuous-time random walks for ordinary and equilibrium cases: facilitation of diffusion, ergodicity breaking and ageing. Phys Chem Chem Phys. 2018;20:2082720848.

47 Sandev T, Metzler R, Chechkin A. From continuous time random walks to the generalized diffusion equation:. Frac Calc Applied Analysis. 2018;21(1):10-28.

48 Vot FL, Abad E, Metzler R, Yuste SB. Continuous time random walk in a velocity field: role of domain growth, Galilei-invariant advection-diffusion, and kinetics of particle mixing. New J Phys. 2020 jul;22(7):073048.

49 Cherstvy AG, Metzler R. Ergodicity breaking, ageing, and confinement in generalized diffusion processes with position and time dependent diffusivity. J Stat Mech. 2015 may;2015(5):P05010.

50 Wang W, Cherstvy AG, Liu X, Metzler R. Anomalous diffusion and nonergodicity for heterogeneous diffusion processes with fractional Gaussian noise. Phys Rev E. 2020 Jul;102:012146.

${ }^{51}$ Reverey JF, Jeon JH, Bao H, Leippe M, Metzler R, Selhuber-Unkel C. Superdiffusion dominates intracellular particle motion in the supercrowded cytoplasm of pathogenic Acanthamoeba castellanii. Sci Rep. 2015;5:2045-2322.

52 Sabri A, Xu X, Krapf D, Weiss M. Elucidating the origin of heterogeneous anomalous diffusion in the cytoplasm of mammalian cells. Phys Rev Lett. 2020 Jul;125:058101.

53 Fulton AB. How crowded is the cytoplasm? Cell. 1982;30:345-347.

${ }^{54}$ Zimmerman SB, Trach SO. Estimation of macromolecule concentrations and excluded volume effects for the cyto- 
plasm of Escherichia coli. J Mol Biol. 1991;222(3):599620 .

55 Zimmerman SB, Minton AP. Macromolecular crowding: biochemical, biophysical, and physiological consequences. Ann Rev Biophys Biomol Struct. 1993;22(1):27-65.

${ }^{56}$ Han J, Herzfeld J. Macromolecular diffusion in crowded solutions. Biophys J. 1993;65:1155-1161.

57 Ellis RJ. Macromolecular crowding: an important but neglected aspect of the intracellular environment. Curr Opin Struct Biol. 2001;11(1):114-119.

58 Ellis RJ. Macromolecular crowding: obvious but underappreciated. Trends Biochem Sci. 2001;26(10):597-604.

59 Minton AP. The influence of macromolecular crowding and macromolecular confinement on biochemical reactions in physiological media. $\mathrm{J}$ Biol Chem. 2001;276(14):10577-10580.

60 Bickmore WA, Sutherland HGE. Addressing protein localization within the nucleus. EMBO J. 2002;21(6):12481254.

61 Golding I, Cox EC. Physical nature of bacterial cytoplasm. Phys Rev Lett. 2006;96:098102.

62 Zhou HX, Rivas G, Minton AP. Macromolecular crowding and confinement: biochemical, biophysical, and potential physiological consequences. Annu Rev Biophys. 2008;37:375-397.

${ }^{63}$ McGuffee SR, Elcock AH. Diffusion, crowding \& protein stability in a dynamic molecular model of the bacterial cytoplasm. PLoS Comput Biol. 2010;6:e1000694.

64 Elcock AH. Models of macromolecular crowding effects and the need for quantitative comparisons with experiment. Curr Opin Struct Biol. 2010;20(2):196-206.

65 Ando T, Skolnick J. Crowding and hydrodynamic interactions likely dominate in vivo macromolecular motion. Proc Natl Acad Sci U S A. 2010;107(43):18457-18462.

${ }^{66}$ Frembgen-Kesner T, Elcock AH. Computer simulations of the bacterial cytoplasm. Biophys Rev. 2013;5:109119.

67 Weiss M. Crowding, diffusion, and biochemical reactions. Intl Rev Cell Mol Biol. 2014;307:383-417.

${ }^{6}$ Parry BR, Surovtsev IV, Cabeen MT, OHern CS, Dufresne ER, Jacobs-Wagner C. The bacterial cytoplasm has glass-like properties and is fluidized by metabolic activity. Cell. 2014;156(1-2):183-194.

${ }^{69}$ Kondrat S, Zimmermann O, Wiechert W, von Lieres E. The effect of composition on diffusion of macromolecules in a crowded environment. Phys Biol. 2015 may;12(4):046003.

70 Rivas G, Minton AP. Macromolecular crowding in vitro, in vivo, and in between. Trends Biochem Sci. 2016;41(11):970-981.

71 André AAM, Spruijt E. Liquid-liquid phase separation in crowded environments. Intl J Mol Sci. 2020;21(16):5908.

72 Sciortino F, Tartaglia P. Glassy colloidal systems. Adv Physics. 2005;54(6-7):471-524.

73 Tolédano P, Neto AMF. Phase Transitions in Complex Fluids. World Scientific; 1998.

74 Tokuyama M. Slow dynamics of equilibrium density fluctuations in suspensions of colloidal hard spheres near the glass transition. Phys Rev E. 2000 Nov;62:R5915-R5918.

75 Hunter GL, Weeks ER. The physics of the colloidal glass transition. Rep Progr Phys. 2012 may;75(6):066501.

76 Braun H, Hentschke R. Phase coexistence for charged soft dumbbell and ionic soft sphere systems via molecular dynamics simulation. Phys Rev E. 2013 Jan;87:012311.

77 Santen L, Krauth W. Absence of thermodynamic phase transition in a model glass former. Nature. 2000;405:550551.

78 Bayer M, Brader JM, Ebert F, Fuchs M, Lange E, Maret $\mathrm{G}$, et al. Dynamic glass transition in two dimensions. Phys Rev E. 2007 Jul;76:011508.

79 Ghosh SK, Cherstvy AG, Metzler R. Non-universal tracer diffusion in crowded media of non-inert obstacles. Phys Chem Chem Phys. 2015;17:1847-1858.

${ }^{80}$ Liao GJ, Klapp SHL. Emergent vortices and phase separation in systems of chiral active particles with dipolar interactions. Soft Matter. 2021;17:6833-6847.

81 Miotto JM, Pigolotti S, Chechkin AV, Roldan-Vargas S. Length scales in Brownian yet non-Gaussian dynamics. Phys Rev X. 2021;11:031002.

${ }^{82}$ Han Y, Alsayed AM, Nobili M, Zhang J, Lubensky TC, Yodh AG. Brownian motion of an ellipsoid. Science. 2006;314(5799):626-630.

${ }^{83}$ Lettinga MP, Grelet E. Self-diffusion of rodlike viruses through smectic layers. Phys Rev Lett. 2007;99(19):197802.

${ }^{84}$ Mukhija D, Solomon MJ. Translational and rotational dynamics of colloidal rods by direct visualization with confocal microscopy. J Colloid Interf Sci. 2007;314(1):98-106.

${ }^{85}$ Han Y, Alsayed A, Nobili M, Yodh AG. Quasi-twodimensional diffusion of single ellipsoids: aspect ratio and confinement effects. Phys Rev E. 2009 Jul;80:011403.

86 Zheng Z, Han Y. Self-diffusion in two-dimensional hard ellipsoid suspensions. J Chem Phys. 2010;133(12):124509.

87 Fakhri N, MacKintosh FC, Lounis B, Cognet L, Pasquali M. Brownian motion of stiff filaments in a crowded environment. Science. 2010;330(6012):1804-1807.

88 Dlugosz M, Antosiewicz JM. Transient effects of excluded volume interactions on the translational diffusion of hydrodynamically anisotropic molecules. J Chem Theory Comput. 2014;10(6):2583-2590.

89 Peng Y, Lai L, Tai YS, Zhang K, Xu X, Cheng X. Diffusion of ellipsoids in bacterial suspensions. Phys Rev Lett. 2016 Feb;116:068303.

90 Bechinger C, Di Leonardo R, Löwen H, Reichhardt C, Volpe G, Volpe G. Active particles in complex and crowded environments. Rev Mod Phys. 2016 Nov;88:045006.

91 Chakrabarty A, Wang F, Sun K, Wei QH. Effects of translation-rotation coupling on the displacement probability distribution functions of boomerang colloidal particles. Soft Matter. 2016;12:4318-4323.

92 Klopp C, Stannarius R, Eremin A. Brownian dynamics of elongated particles in a quasi-two-dimensional isotropic liquid. Phys Rev Fluids. 2017 Dec;2:124202.

93 Shin J, Cherstvy AG, Metzler R. Self-subdiffusion in solutions of star-shaped crowders: non-monotonic effects of inter-particle interactions. New J Phys. 2015;17(11):113028.

94 Shin J, Cherstvy AG, Metzler R. Erratum: Selfsubdiffusion in solutions of star-shaped crowders: nonmonotonic effects of inter-particle interactions [New J. Phys. 17, 113028 (2015)]. New J Phys. 2021 feb;23(2):029601.

95 Segovia-Gutiérrez JP, Escobedo-Sánchez MA, SarmientoGómez E, Egelhaaf SU. Diffusion of anisotropic particles in random energy landscapes-an experimental study. Frontiers Phys. 2020;7:224.

${ }^{96}$ Mazaheri M, Ehrig J, Shkarin A, Zaburdaev V, Sandoghdar V. Ultrahigh-speed imaging of rotational diffusion on 
a lipid bilayer. Nano Lett. 2020;20(10):7213-7219.

97 Skóra T, Vaghefikia F, Fitter J, Kondrat S. Macromolecular crowding: how shape and interactions affect diffusion. The J Phys Chem B. 2020;124(35):7537-7543.

98 Song S, Park SJ, Kim M, Kim JS, Sung BJ, Lee S, et al. Transport dynamics of complex fluids. Proc Natl Acad Sci U S A. 2019;116(26):12733-12742.

99 Nikitin AA, Yurenya AY, Gabbasov RR, Cherepanov VM, Polikarpov MA, Chuev MA, et al. Effects of macromolecular crowding on nanoparticle diffusion: new insights from Mössbauer spectroscopy. J Phys Chem Lett. 2021;12(29):6804-6811.

100 Javanainen M, Ollila OHS, Martinez-Seara H. Rotational diffusion of membrane proteins in crowded membranes. J Phys Chem B. 2020;124(15):2994-3001.

101 Javanainen M, Martinez-Seara H, Kelly CV, Jungwirth P, Fabian B. Anisotropic diffusion of membrane proteins at experimental timescales. J Chem Phys. 2021;155(1):015102.

102 Mayer DB, Sarmiento-Gómez E, Escobedo-Sánchez MA, Segovia-Gutiérrez JP, Kurzthaler C, Egelhaaf SU, et al. Two-dimensional Brownian motion of anisotropic dimers. Phys Rev E. 2021 Jul;104:014605.

103 Roosen-Runge F, Schurtenberger P, Stradner A. Selfdiffusion of nonspherical particles fundamentally conflicts with effective sphere models. J Phys: Cond Matt. 2021 feb;33(15):154002.

104 Berger Bioucas FE, Damm C, Peukert W, Rausch MH, Koller TM, Giraudet C, et al. Translational and rotational diffusion coefficients of gold nanorods dispersed in mixtures of water and glycerol by polarized dynamic light scattering. J Phys Chem B. 2019;123(44):9491-9502.

105 Vasanthi R, Ravichandran S, Bagchi B. Needlelike motion of prolate ellipsoids in the sea of spheres. J Chem Phys. 2001;114(18):7989-7992.

106 Petrelli I, Digregorio P, Cugliandolo LF, Gonnella G, Suma A. Active dumbbells: Dynamics and morphology in the coexisting region. Eur Phys J E. 201825 October; $41: 128$.

107 Heyes DM. Translational and rotational diffusion of rod shaped molecules by molecular dynamics simulations. J Chem Phys. 2019;150(18):184503.

108 Liu Y, Widmer-Cooper A. A dissipative particle dynamics model for studying dynamic phenomena in colloidal rod suspensions. J Chem Phys. 2021;154(10):104120.

109 Nguyen GHP, Wittmann R, Löwen H. Active OrnsteinUhlenbeck model for self-propelled particles with inertia; 2021. Available from: https://arxiv.org/abs/2108. 14005.

110 Roosen-Runge F, Hennig M, Zhang F, Jacobs RMJ, Sztucki M, Schober H, et al. Protein self-diffusion in crowded solutions. Proc Natl Acad Sci U S A. 2011;108(29):1181511820.

111 Heinen M, Zanini F, Roosen-Runge F, Fedunov D, Zhang $\mathrm{F}$, Hennig M, et al. Viscosity and diffusion: crowding and salt effects in protein solutions. Soft Matter. 2012;8:14041419.

112 Kurzthaler C, Leitmann S, Franosch T. Intermediate scattering function of an anisotropic active Brownian particle. Sci Rep. 2016;6:36702.

113 Debnath T, Ghosh PK, Nori F, Li Y, Marchesoni F, Li B. Diffusion of active dimers in a Couette flow. Soft Matter. 2017;13:2793-2799.

114 Sprenger AR, Fernandez-Rodriguez MA, Alvarez L, Isa L,
Wittkowski R, Löwen H. Active Brownian motion with orientation-dependent motility: theory and experiments. Langmuir. 2020;36(25):7066-7073.

115 Verweij RW, Ketzetzi S, de Graaf J, Kraft DJ. Height distribution and orientation of colloidal dumbbells near a wall. Phys Rev E. 2020 Dec;102:062608.

116 Verweij RW, Moerman PG, Huijnen LPP, Ligthart NEG, Chakraborty I, Groenewold J, et al. Conformations and diffusion of flexibly linked colloidal chains. J Phys: Mater. 2021 may;4(3):035002.

117 Mandal S, Kurzthaler C, Franosch T, Löwen H. Crowding-enhanced diffusion: an exact theory for highly entangled self-propelled stiff filaments. Phys Rev Lett. 2020 Sep;125:138002.

118 Rovigatti L, Sciortino F. Self and collective correlation functions in a gel of tetrahedral patchy particles. Mol Phys. 2011;109(23-24):2889-2896.

119 Kondrat S, Popescu MN. Brownian dynamics assessment of enhanced diffusion exhibited by "fluctuating-dumbbell enzymes". Phys Chem Chem Phys. 2019;21(35):1881118815.

${ }^{120}$ Koyano Y, Kitahata H, Mikhailov AS. Diffusion in crowded colloids of particles cyclically changing their shapes. EPL (Europhysics Letters). 2020;128(4):40003.

121 Skora T, Popescu MN, Kondrat S. Conformationchanging enzymes and macromolecular crowding. Phys Chem Chem Phys. 2021;23:9065-9069.

${ }^{122}$ Harth K, Kornek U, Trittel T, Strachauer U, Höme S, Will K, et al. Granular gases of rod-shaped grains in microgravity. Phys Rev Lett. 2013 Apr;110:144102.

123 Brilliantov NV, Pöschel T, Kranz WT, Zippelius A. Translations and rotations are correlated in granular gases. Phys Rev Lett. 2007 Mar;98:128001.

124 Bodrova A, Chechkin AV, Cherstvy AG, Metzler R. Quantifying non-ergodic dynamics of force-free granular gases. Phys Chem Chem Phys. 2015;17:21791-21798.

125 Scholz C, Pöschel T. Velocity distribution of a homogeneously driven two-dimensional granular gas. Phys Rev Lett. 2017 May;118:198003.

${ }^{126}$ Fedorov MV, Kornyshev AA. Ionic liquids at electrified interfaces. Chem Rev. 2014;114:2978.

127 Hayes R, Warr GG, Atkin R. Structure and nanostructure in ionic liquids. Chem Rev. 2015;115(13):6357-6426.

128 Weiss M. Single-particle tracking data reveal anticorrelated fractional Brownian motion in crowded fluids. Phys Rev E. 2013;88:010101.

129 Horton MR, Höfling F, Rädler JO, Franosch T. Development of anomalous diffusion among crowding proteins. Soft Matter. 2010;6:2648-2656.

130 Javanainen M, Hammaren H, Monticelli L, Jeon JH, Miettinen MS, Martinez-Seara H, et al. Anomalous and normal diffusion of proteins and lipids in crowded lipid membranes. Faraday Disc. 2013;161:397-417.

131 Jeon JH, Javanainen M, Martinez-Seara H, Metzler R, Vattulainen I. Protein crowding in lipid bilayers gives rise to non-Gaussian anomalous lateral diffusion of phospholipids and proteins. Phys Rev X. 2016 Apr;6:021006.

132 Yamamoto E, Akimoto T, Mitsutake A, Metzler R. Universal relation between instantaneous diffusivity and radius of gyration of proteins in aqueous solution. Phys Rev Lett. 2021 Mar;126:128101.

133 Jones JE, Chapman S. On the determination of molecular fields. II. From the equation of state of a gas. Proc Royal Soc London Ser A. 1924;106(738):463-477. 
${ }^{134}$ Heitler W, London F. Wechselwirkung neutraler Atome und homöopolare Bindung nach der Quantenmechanik. Z für Physik. 1927;44:455472.

135 Lennard-Jones JE. The electronic structure of some diatomic molecules. Trans Faraday Soc. 1929;25:668-686.

136 Buckingham RA, Lennard-Jones JE. The classical equation of state of gaseous helium, neon and argon. Proc Royal Soc London Ser A. 1938;168(933):264-283.

137 Andersen HC, Weeks JD, Chandler D. Relationship between the hard-sphere fluid and fluids with realistic repulsive forces. Phys Rev A. 1971 Oct;4:1597-1607.

138 Weeks JD, Chandler D, Andersen HC. Role of repulsive forces in determining the equilibrium structure of simple liquids. J Chem Phys. 1971;54(12):5237-5247.

139 Chandler D, Weeks JD, Andersen HC. Van der Waals picture of liquids, solids, and phase transformations. Science. 1983:220(4599):787-794.

140 Balakrishnan V. All about the Dirac delta function(?). Resonance. 2003;8:48-58.

141 Fejes TL. Über einen geometrischen Satz. Mathematische Zeitschrift. 1940;46:83-85.

142 Fejes TL. Über die dichteste Kreislagerung und dünnste Kreisüberdeckung. Commentarii Mathematici Helvetici. 1949;23:342-349.

143 Penrose O, Stell G. Close to close packing. J Stat Phys. 2000;100:89-95.

144 Wilken S, Guerra RE, Levine D, Chaikin PM. Random close packing as a dynamical phase transition. Phys Rev Lett. $2021 \mathrm{Jul} ; 127: 038002$.

145 Vladimirsky V, Terletzky YA. Hydrodynamic theory of translational Brownian motion. Zh Eksp Teor Fiz. 1945;15:258-263.

146 Batchelor GK. Brownian diffusion of particles with hydrodynamic interaction. J Fluid Mech. 1976;74(1):129.

147 Hinch EJ. Application of the Langevin equation to fluid suspensions. J Fluid Mech. 1975;72(3):499511.

148 Elgeti J, Winkler RG, Gompper G. Physics of microswimmers - single particle motion and collective behavior: a review. Rep Progr Phys. 2015 apr;78(5):056601.

149 Zöttl A, Stark H. Emergent behavior in active colloids. J Phys: Cond Matt. 2016 may;28(25):253001.

150 Allen MP, Tildesley DJ. Computer simulations of liquids. Oxford: Clarendon; 1987.

151 Bickmore WA, Sutherland HGE. Addressing protein localization within the nucleus. EMBO J. 2002;21(6):12481254.

152 Bian X, Kim C, Karniadakis GE. 111 years of Brownian motion. Soft Matter. 2016;12:6331-6346.

153 Landau LD, Lifschitz JM, Pitajewski LP. Lehrbuch der theoretischen Physik: V. Statistische Physik [Teil 1]. Akademie-Verlag Berlin; 1979.

154 Leitmann S, Franosch T. Time-dependent fluctuations and superdiffusivity in the driven lattice Lorentz gas. Phys Rev Lett. 2017 Jan;118:018001.

155 Petersen CF, Franosch T. Anomalous transport in the soft-sphere Lorentz model. Soft Matter. 2019;15:39063913.

156 Cherstvy AG, Thapa S, Mardoukhi Y, Chechkin AV, Metzler R. Time averages and their statistical variation for the Ornstein-Uhlenbeck process: Role of initial particle distributions and relaxation to stationarity. Phys Rev E. 2018 Aug;98:022134.

157 Boltzmann L. Ueber die Eigenschaften monocyklischer und anderer damit verwandter Systeme. J reine und ange- wandte Mathematik. 1885;98:68-94.

158 Berezhkovskii AM, Dagdug L, Bezrukov SM. Discriminating between anomalous diffusion and transient behavior in microheterogeneous environments. Biophys J. 2014;106(2):L09-L11.

159 Qiu X, Wu XL, Xue JZ, Pine DJ, Weitz DA, Chaikin PM. Hydrodynamic interactions in concentrated suspensions. Phys Rev Lett. 1990 Jul;65:516-519.

160 van Megen W, Underwood SM. Tracer diffusion in concentrated colloidal dispersions. III. Mean squared displacements and self-diffusion coefficients. J Chem Phys. 1989;91(1):552-559.

161 Banchio AJ, Nägele G. Short-time transport properties in dense suspensions: From neutral to charge-stabilized colloidal spheres. J Chem Phys. 2008;128(10):104903.

162 Quesada-Pérez M, Maroto-Centeno JA, Ramos-Tejada MdM, Martn-Molina A. Universal description of steric hindrance in flexible polymer gels. Phys Chem Chem Phys. 2021;23:14997-15002.

163 Santen L, Krauth W. Absence of thermodynamic phase transition in a model glass former. Nature. 2000;405:550551.

164 Tokuyama M, Oppenheim I. Dynamics of hard-sphere suspensions. Phys Rev E. 1994 Jul;50:R16-R19.

165 Tokuyama M, Oppenheim I. On the theory of concentrated hard-sphere suspensions. Physica A. 1995;216(1):85-119.

166 Franosch T, Fuchs M, Götze W, Mayr MR, Singh AP. Asymptotic laws and preasymptotic correction formulas for the relaxation near glass-transition singularities. Phys Rev E. 1997 Jun;55:7153-7176.

167 Weysser F, Hajnal D. Tests of mode-coupling theory in two dimensions. Phys Rev E. 2011 Apr;83:041503.

168 Beenakker CWJ, Mazur P. Self-diffusion of spheres in a concentrated suspension. Physica A. 1983;120(3):388410.

169 Beenakker CWJ, Mazur P. Diffusion of spheres in a concentrated suspension II. Physica A. 1984;126(3):349-370.

170 Medina-Noyola M. Long-time self-diffusion in concentrated colloidal dispersions. Phys Rev Lett. 1988 Jun;60:2705-2708.

171 Zhu JX, Durian DJ, Müller J, Weitz DA, Pine DJ. Scaling of transient hydrodynamic interactions in concentrated suspensions. Phys Rev Lett. 1992 Apr;68:2559-2562.

172 Brady JF. The rheological behavior of concentrated colloidal dispersions. J Chem Phys. 1993;99(1):567-581.

173 Beenakker CWJ. The effective viscosity of a concentrated suspension of spheres (and its relation to diffusion). Physica A. $1984 ; 128(1): 48-81$.

174 Tokuyama M. Effective diffusion model on Brownian dynamics of hard-sphere colloidal suspensions. Physica A. 1999;265(3):333-340.

175 Watzlawek M, Nägele G. Self-diffusion coefficients of charged particles: Prediction of nonlinear volume fraction dependence. Phys Rev E. 1997 Jul;56:1258-1261.

176 Sun J, Weinstein H. Toward realistic modeling of dynamic processes in cell signaling: quantification of macromolecular crowding effects. J Chem Phys. 2007;127(15):155105.

177 Hannam SDW, Daivis PJ, Bryant G. Dynamics of a model colloidal suspension from dilute to freezing. Phys Rev E. 2016 Jul;94:012619.

178 van Blaaderen A, Peetermans J, Maret G, Dhont JKG. Long-time self-diffusion of spherical colloidal particles measured with fluorescence recovery after photobleaching. 
The Journal of Chemical Physics. 1992;96(6):4591-4603.

179 Makuch K, Heinen M, Abade GC, Nägele G. Rotational self-diffusion in suspensions of charged particles: simulations and revised Beenakker-Mazur and pairwise additivity methods. Soft Matter. 2015;11:5313-5326.

180 Banchio AJ, Heinen M, Holmqvist P, Nägele G. Shortand long-time diffusion and dynamic scaling in suspensions of charged colloidal particles. J Chem Phys. 2018;148(13):134902.

181 Gao Y, Chen Y, Ji X, He X, Yin Q, Zhang Z, et al. Controlled intracellular release of doxorubicin in multidrug-resistant cancer cells by tuning the shell-pore sizes of mesoporous silica nanoparticles. ACS Nano. 2011;5(12):9788-9798.

182 Díez Fernández A, Charchar P, Cherstvy AG, Metzler R, Finnis MW. The diffusion of doxorubicin drug molecules in silica nanoslits is non-Gaussian, intermittent and anticorrelated. Phys Chem Chem Phys. 2020;22:27955-27965.

183 Di Pierro M, Potoyan DA, Wolynes PG, Onuchic JN. Anomalous diffusion, spatial coherence, and viscoelasticity from the energy landscape of human chromosomes. Proc Natl Acad Sci U S A. 2018;115(30):7753-7758.

184 Goychuk I. In: Viscoelastic Subdiffusion: Generalized Langevin Equation Approach. John Wiley Sons, Ltd; 2012. p. 187-253.

185 Landau LD, Lifschitz JM. Lehrbuch der theoretischen Physik: I. Mechanik. Akademie-Verlag Berlin; 1990.

186 Bakalis E, Höfinger S, Venturini A, Zerbetto F. Crossover of two power laws in the anomalous diffusion of a two lipid membrane. J Chem Phys. 2015;142(21):215102.

187 Sandev T, Metzler R, Tomovski Z. Velocity and displacement correlation functions for fractional generalized Langevin equations. Fract Calc Appl Analysis. 2012;15(3):426-450.

188 Vrentas JS, Vrentas CM. Viscoelastic diffusion. J Polymer Sci B. 2001;39(13):1529-1547.

189 Thapa S, Lomholt MA, Krog J, Cherstvy AG, Metzler R. Bayesian analysis of single-particle tracking data using the nested-sampling algorithm: maximum-likelihood model selection applied to stochastic-diffusivity data. Phys Chem Chem Phys. 2018;20:29018-29037.

190 Cherstvy AG, Thapa S, Wagner CE, Metzler R. NonGaussian, non-ergodic, and non-Fickian diffusion of tracers in mucin hydrogels. Soft Matter. 2019;15:2526-2551.

191 Reina F, Wigg JMA, Dmitrieva M, Lefebvre J, Rittscher J, Eggeling C. TRAIT2D: a software for quantitative qnalysis of single particle diffusion data. F1000Research. 2021;10:838. Available from: https://doi.org/10. 12688/f1000research.54788.1.

192 Granik N, Weiss LE, Nehme E, Levin M, Chein M, Perlson E, et al. Single-particle diffusion characterization by deep learning. Biophys J. 2019;117(2):185-192.

193 Muñoz-Gil G, Garcia-March MA, Manzo C, MartínGuerrero JD, Lewenstein M. Single trajectory characterization via machine learning. New J Phys. 2020 jan;22(1):013010.

194 Muñoz-Gil G, Volpe G, Garcia-March MA, Aghion E, Argun A, Hong CB, et al.. Objective comparison of methods to decode anomalous diffusion; 2021. Available from: https://arxiv.org/abs/2105.06766.

195 Janczura J, Kowalek P, Loch-Olszewska H, Szwabiński J, Weron A. Classification of particle trajectories in living cells: Machine learning versus statistical testing hypoth- esis for fractional anomalous diffusion. Phys Rev E. 2020 Sep;102:032402.

196 Gajowczyk M, Szwabinski J. Detection of anomalous diffusion with deep residual networks. Entropy. 2021;23(6).

197 Reyes A, Viera-Lopez G, Morgado-Vega JJ, Altshuler E. yupi: Generation, tracking and analysis of trajectory data in Python; 2021. Available from: https://arxiv.org/ abs/2108.06340.

198 Pinholt HD, Bohr SSR, Iversen JF, Boomsma W, Hatzakis NS. Single-particle diffusional fingerprinting: A machine-learning framework for quantitative analysis of heterogeneous diffusion. Proc Natl Acad Sci U S A. 2021;118(31):e2104624118.

199 Caetano DLZ, Metzler R, Cherstvy AG, de Carvalho SJ. Adsorption of lysozyme into a charged confining pore. bioRxiv. 2021;Available from: https://www.biorxiv. org/content/earlv/2021/07/12/2021.07.11.451934.

200 Simunovic M, Srivastava A, Voth GA. Linear aggregation of proteins on the membrane as a prelude to membrane remodeling. Proc Natl Acad Sci U S A. 2013;110(51):2039620401.

201 Cherstvy AG, Petrov EP. Modeling DNA condensation on freestanding cationic lipid membranes. Phys Chem Chem Phys. 2014;16:2020-2037.

202 Simunovic M, Voth GA. Membrane tension controls the assembly of curvature-generating proteins. Nature Comm. 2015;6:7219.

203 Voth GA, Callan-Jones A, Bassereau P. When physics takes over: BAR proteins and membrane curvature. Quant Cell Biol. 2015;25:780-792.

204 Ghosh SK, Cherstvy AG, Petrov EP, Metzler R. Interactions of rod-like particles on responsive elastic sheets. Soft Matter. 2016;12:7908-7919.

205 Petrova AB, Herold C, Petrov EP. Conformations and membrane-driven self-organization of rodlike fd virus particles on freestanding lipid membranes. Soft Matter. 2017;13:7172-7187.

206 Spohr HV, Patey GN. Structural and dynamical properties of ionic liquids: The influence of ion size disparity. J Chem Phys. 2008;129(6):064517.

207 Caetano DLZ, Bossa GV, de Oliveira VM, Brown MA, de Carvalho SJ, May S. Differential capacitance of an electric double layer with asymmetric solvent-mediated interactions: mean-field theory and Monte Carlo simulations. Phys Chem Chem Phys. 2017;19:23971-23981.

208 Yang G, Oh H, Chanthad C, Wang Q. Dumbbell-shaped octasilsesquioxanes functionalized with ionic liquids as hybrid electrolytes for lithium metal batteries. Chemistry of Materials. 2017;29(21):9275-9283.

209 Dussi S, Rovigatti L, Sciortino F. On the gas-liquid phase separation and the self-assembly of charged soft dumbbells. Mol Phys. 2013;111(22-23):3608-3617.

210 Budkov YA. Statistical theory of fluids with a complex electric structure: Application to solutions of soft-core dipolar particles. Fluid Phase Equil. 2019;490:133-140.

211 Budkov YA. Nonlocal statistical field theory of dipolar particles forming chain-like clusters. J Mol Liq. 2019;276:812-818.

212 Budkov YA, Kalikin NN, Kolesnikov AL. Molecular theory of the electrostatic collapse of dipolar polymer gels. Chem Comm. 2021;57:3983-3986. 